\title{
Flow pattern assessment in tubes of reciprocating scraped surface heat exchangers
}

\author{
J.P. Solano ${ }^{* a}$, A. García ${ }^{a}$, P.G. Vicente ${ }^{\mathrm{b}}$, A. Viedma ${ }^{\mathrm{a}}$ \\ ${ }^{a}$ Universidad Politécnica de Cartagena, Departamento de Ingeniería Térmica y de Fluidos, \\ Campus Muralla del Mar, 30202 Cartagena, Spain \\ ${ }^{b}$ Universidad Miguel Hernández, Departamento de Ingeniería de Sistemas Industriales, Avenida \\ de la Universidad, 03202 Elche, Spain
}

\begin{abstract}
Flow pattern in the tubes of an innovative scraped surface heat exchanger with reciprocating scrapers has been experimentally investigated. The scraper consists of a concentric rod inserted in each tube of the heat exchanger, mounting an array of semicircular plugs that fit the inner tube wall. A hydraulic piston provides the scraper with constant-velocity reciprocating motion. Phase-averaged velocity fields have been obtained with PIV technique for both scraping semi-cycles, with special emphasis on the effect of the scraping velocity (velocity ratio) and Reynolds number. Visualization results have been contrasted with experimental data on Fanning friction factor, obtaining a clear relation between flow patterns, pressure drop augmentation and turbulence promotion. CFD simulations for quasi-steady laminar flow provide a further insight into the relation of the flow structures with wall shear stress, and the contribution of pressure forces to global head losses, for each scraping semi-cycle.
\end{abstract} Key words: SSHE, PIV, flow pattern, friction factor, CFD

\footnotetext{
${ }^{*}$ Corresponding autor

Email address: juanp.solano@upct.es (J.P. Solano)
} 


\section{Nomenclature}

$\begin{array}{ll}A & \text { annular cross-section }\left(\mathrm{m}^{2}\right) \\ D & \text { tube inner diameter }(\mathrm{m}) \\ D_{h} & \text { hydraulic diameter }(\mathrm{m}) \\ d & \text { rod diameter }(\mathrm{m}) \\ F & \text { force over a scraper pitch }(\mathrm{N}) \\ \dot{m} & \text { mass flow rate }\left(\mathrm{kg} \mathrm{s}^{-1}\right) \\ P & \text { pitch of the insert devices }(\mathrm{m}) \\ \Delta p & \text { pressure drop across the test section }(\mathrm{Pa}) \\ S & \text { scraping amplitude }(\mathrm{m}) \\ T & \text { temperature }(\mathrm{K}) \\ v & \text { velocity }\left(\mathrm{m} \mathrm{s}^{-1}\right)\end{array}$

Dimensionless groups

$f_{h} \quad$ Fanning friction factor

$\mathrm{Re}_{h} \quad$ Reynolds number

$\omega \quad$ velocity ratio, $v_{p} / v_{f}$

$\mathrm{c}_{F} \quad$ force coefficient, $F / \frac{1}{2} \rho v_{f}^{2} A$

$\mathrm{C}_{f} \quad$ skin friction coefficient, $\tau_{w} / \frac{1}{2} \rho v_{f}^{2}$

Greek symbols

$\beta \quad$ blockage parameter $\left(v_{f}-v_{p}\right) / v_{f}(-)$

$\Gamma \quad$ numerical surface

$\kappa \quad$ radii ratio, $\mathrm{d} / \mathrm{D}(-)$

$\mu \quad$ dynamic viscosity $(\mathrm{Pa} \cdot \mathrm{s})$ 


$\begin{array}{ll}\Omega & \text { numerical volume } \\ \rho & \text { fluid density }\left(\mathrm{kg} \mathrm{m}^{-3}\right) \\ \tau & \text { wall shear stress }\left(\mathrm{N} \mathrm{m}^{-2}\right) \\ & \\ \text { Subscripts } & \\ \text { cc } & \text { counter-current } \\ \text { eq } & \text { co-current } \\ f & \text { fluid } \\ \text { in } & \text { tube inlet } \\ m e d & \text { average } \\ \text { out } & \text { tube outlet } \\ p & \text { scraper } \\ s & \text { smooth tube } \\ w & \text { tube wall } \\ z & \text { axial direction }\end{array}$

\section{Introduction}

Scraped surface heat exchangers (SSHE) are commonly employed for heat transfer and crystallization processes in the food, chemical and pharmaceutical industries. These mechanically assisted devices are specially suited for products that are viscous, sticky or that contain particulate matter. During operation, the product is brought in contact with a heat transfer surface that is continuously scraped, thereby exposing the surface to the passage of untreated product [1].

High heat transfer coefficients are achieved because the boundary layer is continuously replaced by fresh material, while heat transfer surfaces remain clean. In 
addition to maintain high and uniform heat exchange, the scraper blades also provide simultaneous mixing and agitation, of utmost importance for laminar flow heat transfer enhancement [2]. Most commercial designs have a rotating shaft in the center with the product being pumped through the annular gap between the shaft and the outer cylindrical heat transfer tube. Flow and shearing profiles have been widely studied since the first works of Trommelen and Beek [3]. Influence of rotational speed and presence of blades has been analyzed in the remarkable works of Stranzinger et al. [4] and Dumont et al. [5], clarifying three different regimes characterized by pure shear flow, steady toroidal vortices and unstable, wavy vortices. Extensive reviews covering design aspects, heat transfer characteristics and power consumption of SSHE's can be found in Harrod [6] and Rao and Hartel [1].

This work presents an innovative concept of scraped surface heat exchanger. Within each tube is a concentric reciprocating rod, mounting an array of semi-circular elements with a pitch $\mathrm{P}=5 \mathrm{D}$ (see Fig. 1). These elements fit the internal diameter of the tubes. During the reciprocating motion, they scrape the inner tube wall. Additionally, the movement of the insert device generates macroscopic displacements of the flow, that continuously mix core regions with peripheral flow. As a result of the mentioned features, the reciprocating scraped surface heat exchanger (RSSHE) provides high overall heat transfer coefficients, and prevents down time for cleaning operations. Depending on the severity of the fouling phenomenon, the scraper can be either activated intermittently, or move continuously with adjustable scraping frequencies.

Commercially available versions of this RSSHE are manufactured by HRS-Spiratube S.L. under the brand UNICUS Dynamic Heat Exchanger, and by Alfa Laval Inc, with the brand Viscoline Dynamic Unit. Along with the industrial evidence of fouling reduction, the manufacturers claim for its higher heat transfer area, smaller number 
of shutdowns, lower induced shear stress in the product, and particle integrity in food applications, compared to rotating scraped surface heat exchangers. RSSHE's are progressively being introduced in the food industry, wastewater treatment processes, production of second-generation biofuels, etc.

The present work is devoted to the analysis of the flow pattern and the friction characteristics in tubes of reciprocating scraped surface heat exchangers. Particle Image Velocimetry (PIV) technique is employed for obtaining the two-dimensional velocity field in the symmetry plane of the tube in laminar regime. The separate performance of the device in the two scraping semi-cycles is considered in the analysis, thus defining counter-current and co-current stages. Flow patterns existing for different velocity ratios $\omega$ are identified, establishing as reference condition the per-

formance of the still scraper. The influence of Reynolds number on the flow features is assessed.

Experimental results of Fanning friction factor for a wide range of Reynolds numbers and velocity ratios are contrasted with the visualization data, for both scraping semicycles. The contribution of the flow structures to the local shear stress is briefly analyzed with the numerical simulation tool Fluent, complementing the experimental data and providing further information towards the holistic understanding of the physical flow nature.

\section{Experimental program}

\subsection{Visualization Facility}

The facility depicted in Fig. 2 was built in order to study the flow pattern induced by a wide variety of insert devices in round tubes [7]. The main section consists of a $32 \mathrm{~mm}$ diameter acrylic tube installed between two reservoir tanks, that stabilize 
the flow. The flow temperature is regulated by an electric heater and a thermostat placed in the upper reservoir tank. The flow is impelled from the lower calm deposit to the upper one by a gear pump, which is adjusted by a frequency converter. By using mixtures of water and propylene-glycol at temperatures from $20^{\circ} \mathrm{C}$ to $60^{\circ} \mathrm{C}$, Reynolds numbers between 100 and 20000 can be obtained. The tests presented in this work were carried out employing a mixture of $90 \%$ propylene-glycol and $10 \%$ water, at temperatures from $25^{\circ} \mathrm{C}$ to $50^{\circ} \mathrm{C}$, yielding Reynolds numbers in the range from 36 to 378 .

The test section has been placed downstream five scraper pitches (25D), thus ensuring periodic flow conditions. To improve optical access in this section, a flat-sided acrylic box was placed around. The box was filled with the same test fluid that flows through the test section. Particle Image Velocimetry (PIV) has been employed for flow visualization.

PIV is a well-known technique to obtain global velocity information, instantaneously and with high accuracy [8]. In these experiments, planar slices of the flow field containing the symmetry plane of the inserted device were illuminated. The flow was seeded with polyamide particles of $57 \mu \mathrm{m}$ mean diameter. The camera viewed the illuminated plane from an orthogonal direction and recorded particle images at two successive instants in time in order to extract the velocity over the planar two-dimensional domain. 2D velocity fields along a scraper pitch were assembled to provide an overall insight of the flow structure.

The spatial resolution of the measurement is $110 \mu \mathrm{m} /$ pixel. A $1 \mathrm{~mm}$ thick light sheet is created by a pulsating diode laser of $808 \mathrm{~nm}$ wavelength. A computer synchronizes the camera shutter opening and the laser shot at sampling frequencies between 250 and $500 \mathrm{~Hz}$, most appropriate for the test conditions. The sketch of the measurement arrangement is similar to that employed by [7]. 
The high speed camera of the PIV system was employed separately to measure the piston velocity during a characteristic scraping cycle, as shown in Fig. 3. During both semi-cycles the piston moves with constant velocity, but transient disturbances shortly occur when the piston motion reverses. Velocity levels and duration of both semi-cycles are nearly identical. When the piston moves in the same direction of the flow $\left(v_{p}>0\right)$, the semi-cycle is called co-current, whereas the movement of the piston in opposite direction to the flow $\left(v_{p}<0\right)$ is called counter-current.

As the hydraulic piston moves with constant velocity, quasi-steady velocity profiles inside the tube can be considered from a moving reference frame. Therefore, for each working condition, two velocity fields - co-current and counter-current- will be considered to fully characterize the flow pattern during the scraping cycle. Phaseaveraged PIV measurements are performed separately for each semi-cycle, following the methodology sketched in Fig. 4. A photosensor (1) is located in the bottom of the acrylic tube. The end of the moving rod (2) activates this sensor for a certain duration over the cycle, covering the reversal from counter-current to co-current motion. The appropriate configuration of the photo-sensor output (normally closed in counter-current measurements, and normally open in co-current tests), and the subsequent activation of a trigger allows capturing a pair of images at constant phase for each complete scraping cycle. Ensembled-average of 50 pairs of images were done for obtaining the velocity field over the symmetry plane of the tube in both semicycles.

A non-dimensional parameter, the velocity ratio $\omega$, is introduced to account for the movement of the device inside the tube:

$$
\omega=\frac{v_{p}}{v_{f}}
$$

The velocity ratio will be positive for co-current conditions, and negative for counter- 
current mode. The derivation of $v_{p}$ is performed by means of the cycle duration measurement, $\Delta t$, obtained with a pulse timer, provided that the position of the endof-stroke switches ensure a known scraping amplitude $S=2 P$. Fluid velocity $v_{f}$ was derived from flow rate measurements obtained with an electromagnetic flowmeter. A different formulation of the velocity ratio can be expressed as:

$$
\beta=\frac{v_{f}-v_{p}}{v_{f}}
$$

being $\beta$ the blockage parameter, $\beta=1-\omega$, that can be computed in order to assess the blockage effect $(\beta>0)$ or dragging effect $(\beta<0)$ of the scraper on the flow. Image processing was carried out with the software 'vidPIV', that applies a crosscorrelation algorithm between consecutive images. The interrogation window size used for PIV processing was $32 \times 32$ pixels, with a $50 \%$ overlap. Subsequent adaptive cross-correlation algorithm [9] was applied with an interrogation window size of $16 \times$ 16 pixels and $50 \%$ overlap. To obtain a clear velocity field, after the images were correlated, a global and a local filter were applied to remove outliers. The resulting vectors were averaged over fifty realizations. Using the standard formula for 20-to-1

odds, $\pm 1.96 \sigma N^{-0.5}$, the uncertainty in PIV measurements is about $3 \%$. Taking into account the uncertainty in the computation of pixels displacements, the final uncertainty in PIV measurements rises to $5 \%$.

\subsection{Friction factor measurement tests}

A schematic diagram of the experimental setup is shown in Fig. 5. The test section consists of a $20 \times 1 \mathrm{~mm}$ smooth tube where the scraper is inserted. A secondary circuit is used for regulating the working fluid temperature. Propylene-glycol is employed as working fluid. 
Pressure measurement ports were separated a distance $l_{p}=20 \times P$, and consisted of four pressure holes peripherally spaced by $90^{\circ}$. Test section was preceded by a development region of $l_{e}=6 \times P$ length, in order to establish periodic flow conditions. The scraping amplitude of the insert device was $S=2 P$, so that this development region reduced to $l_{e}=4 P$ for the end-of-stroke co-current motion.

Fluid inlet and outlet temperatures, $T_{\text {in }}$ and $T_{\text {out }}$ were measured by submerged type RTDs (Resistance Temperature Detector). Since test and developing flow regions were insulated to ensure isothermal conditions, the fluid temperature was calculated by $T_{f}=\left(T_{\text {in }}+T_{\text {out }}\right) / 2$.

Single gauge piezorresistive transducers were coupled to each end of the pressure test section, to acquire the time-dependent pressure drop occuring during the dynamic performance of the scraper. A highly accurate, differential pressure sensor was additionally employed for the motionless tests.

Unsteady pressure drop across the tube was computed as the difference between both signals obtained with the piezorresistive transducers, $\Delta p(t)=p_{1}(t)-p_{2}(t)$. A detail of this signal, for a characteristic scraping cycle, is shown in Fig. 6. The periodicity of the signal corresponds to the scraping frequency. During the countercurrent semi-cycle, a higher pressure level is observed in the tube. When the scraper moves in co-current direction, the pressure level decreases. As a result, the pressure drop signal approaches to a square wave, influenced by the constant velocity motion of the piston.

Signal is split into counter-current and co-current frames in order to derive the separate pressure drop occurring in the tube during each semi-cycle. Pressure drop is time-averaged over a sufficient number of cycles, typically $n>50$, along $\Delta \mathrm{t}_{m}$ intervals centered in each semi-cycle (see Fig. 6).

Fanning friction factor for each semi-cycle, based on the hydraulic diameter of the an- 
nular section, was determined from mass flow rate measurements and time-averaged pressure drop results as:

$$
f_{h}=\frac{\overline{\Delta p}}{l_{p}} \frac{\rho \pi^{2}(D+d)^{2} D_{h}^{3}}{32 \dot{m}^{2}}
$$

Experimental uncertainty was calculated by following the "Guide to the expression of uncertainty in measurement" published by ISO [10]. Uncertainty calculations based on a 95 percent confidence level showed maximum values of $4 \%$ for Reynolds number, $3 \%$ for friction factor in motionless conditions, $8.5 \%$ for friction factor in dynamic conditions, and $7 \%$ for velocity ratio.

\section{Numerical model and mesh}

The geometry of the computational model was created using the software Gambit. It consists of an annular duct to which semi-circular plugs are substracted every $P / 2$ distance, which constitutes the fluid volume of the inserted tube. The scraper extends over four pitches, being the flow solution in the third one subjected to analysis. This ensured periodic flow conditions in the entrance and flow free of outlet effects. The geometrical symmetry of the problem proved to ensure also flow symmetry for the range of Reynolds number under study. Thus, only half domain was modelled.

Structured, hexahedral mesh was employed (see Fig. 7). A double compression ratio was introduced in both sides of the plugs, where greater variations of the flow pattern exists due to the geometry constriction, and in radial direction, to ensure better solution where higher velocity gradients where expected.

The numerical problem was considered quasi-steady from a moving reference frame located on the scraper. This modeling assumption is supported by two main features of the flow in tubes of RSSHE's. The first feature comes from the experimental evidence of constant velocity motion of the scraper during both semi-cycles, as demon- 
strated in the instantaneous scraping velocity profile depicted in Fig. 3. Besides, the flow features at the commencement of each semi-cycle present a short duration transient, being the quasi-steady flow pattern arisen representative of the most part of each scraping semi-cycle. This short-duration phenomenon was observed during the visualization campaign, and numerically quantified as $1 / 4$ of the semi-cycle duration in the most unfavourable situation $(\omega= \pm 2)$.

To account for this solution strategy, Multiple Reference Frame (MRF) model [11] was employed in the finite volume code FLUENT (commercially available software, version 6.3). Absolute velocities are used in momentum equations as dependent variables, yielding to the absolute formulation of the governing equations of the problem:

$$
\begin{aligned}
& \nabla \cdot \overrightarrow{v^{\prime}}=0 \\
& \nabla \cdot\left(\rho \overrightarrow{v^{\prime}} \vec{v}\right)=-\nabla p+\nabla \overline{\bar{\tau}}
\end{aligned}
$$

where

$$
\overrightarrow{v^{\prime}}=\vec{v}-\overrightarrow{v_{p}}
$$

The next boundary conditions are imposed through the MRF model:

$$
\begin{aligned}
& \vec{v}\left(\Gamma_{i n}\right)=\overrightarrow{v_{i n}}-\overrightarrow{v_{p}} \\
& \vec{v}\left(\Gamma_{w}\right)=-\overrightarrow{v_{p}}
\end{aligned}
$$

Control-volume storage scheme was employed where all variables were stored at the cell center. Second order upwind scheme was used in order to interpolate the face 
values of computed variables. Implicit segregated solver solved the governing equations sequentially. In this study, pressure-velocity coupling algorithm SIMPLE was used.

The Grid Convergence Index (GCI) method has been employed to compute the numerical uncertainty of the simulations (Celik et al. [12]). Systematic grid refinement was assessed with three significantly different sets of grids, the finest of which is employed to obtain the final results (Fig. 7). Maximum values of GCI of $3.49 \%$ have been found for the computation of the velocity, $0.85 \%$ for the pressure and $2.47 \%$ for the derivation of the force coefficient. A summary of the results for $\mathrm{Re}_{h}=80$ and $\omega= \pm 0.5, \pm 1$ and \pm 2 is reported in Table 1.

\section{Flow description}

During the counter-current semi-cycle, the scraper imposes a positive blockage to the flow, $\beta>0$, with independence of the velocity ratio. This positive blockage is also found for the motionless scraper $(\omega=0)$, and comprises a common velocity pattern for all these working conditions.

Fig. 8 depicts the non-dimensional velocity field, $\vec{v} / v_{\text {med }}$ in the symmetry plane of the scraper, for $\operatorname{Re}_{h}=40$ and $\omega=-1$. This condition is sufficiently representative of the positive blockage phenomenon. A horizontal plane containing the tube axis also divides the visualization field into bottom and top sections, with odd-symmetry characteristics.

Following the sequence of the flow direction (left to right), a region of low velocity is found in the top section, downstream the first plug. The flow vectors enclosed to the picture move backwards, which is associated to flow separation and recirculation. Afterwards, a high velocity region grows along the streamwise direction, and finally impacts against the third plug, generating a transverse vortex. 
The mechanism yielding to the appeareance of these structures is related to the meandering path of the flow: the presence of the plugs every $P / 2$ distance forces the flow to sharply turn and move towards the section not affected by the blockage. The resulting deviation induces a local velocity increase, as the flow area is approximately reduced by one half. The main stream reattaches to the wall and proceeds towards the tube. As this stream expands to the annular section, two counter-rotating recirculation bubbles are created behind the plugs. These bubbles converge in the symmetry plane, where the local structure with low velocity, reversed flow vectors are measured. The reattached flow finally impacts against the next plug, creating a transverse vortex rolling over a curved axis parallel to the front side of the plug.

\subsection{Counter-current mode}

Fig. 9 represents the velocity field in the symmetry plane of the inserted tube at Reynolds number $\operatorname{Re}_{h}=80$, for the limiting condition of motionless scraper $(\omega=0)$, and for velocity ratios $\omega=-0.5,-1$ and -2 . This picture allows analyzing the effect of the scraper movement on the flow structures.

Two main features of the recirculation bubbles are enhanced during the countercurrent motion: the characteristic size and the local velocity level. The length of these bubbles reaches up to $L / P \approx 0.3$ in dynamic conditions. This value appears to be asymptotic, as the mean flow deviates along a characteristic tube stream to overcome the next plug. Additionally, the local velocity levels of the reversed flow are higher than those found for the motionless condition $\left(v / v_{\text {med }} \approx 0.1\right)$, owing to the higher static pressure deficit induced by the scraper movement. Values of the order of $v / v_{\text {med }} \approx 1$ are reported for $\omega=-0.5$, and $v / v_{\text {med }} \approx 3$ for $\omega=-2$. Higher wall shear rates are thus expected downstream the plugs. The dynamics of the recirculation bubbles convey colder fluid from the center of the tube towards the wall, and will 
eventually contribute to increase the local heat transfer coefficient in this region. The transverse vortex is also depicted in Fig. 10, and contrasted with the motionless solution. It is clear that the movement of the plugs promotes the appearance of the vortex, which maintains a similar shape and size, as shown for $\mathrm{Re}_{h}=80$ and the tested velocity ratios. Local velocities are in all dynamic conditions much higher than those found for $\omega=0$, of the order of $v / v_{\text {med }} \approx 2.5$.

Another important feature amplified by the counter-current working mode is the global velocity level reached in the high velocity region. Velocity fields in Fig. 9 allow identifying this evolution, which reaches values as high as $v_{\text {max }} / v_{\text {med }} \approx 4$ for $\omega=-0.5$ and $v_{\text {max }} / v_{\text {med }} \approx 7$ for $\omega=-2$. This is contrasted with the lower level, $v_{\text {max }} / v_{\text {med }} \approx 3$, reported for $\omega=0$. A more detailed analysis of the velocity profile allows stating the influence of Reynolds number on the flow nature. Fig. 11 presents the velocity profile in a section located at a distance $P / 5$ of the front side of the plug, for $\omega=0,-0.5,-1$ and -2 . Results are contrasted with the analytical solution for the concentric annuli with counter-current moving core and corresponding velocity ratio:

$$
\frac{v}{v_{m e d}}=\frac{1-\omega\left(\frac{1}{2 \ln (1 / \kappa)}-\frac{\kappa^{2}}{1-\kappa^{2}}\right)}{\frac{1}{2}\left(\frac{1-\kappa^{4}}{1-\kappa^{2}}-\frac{1-\kappa^{2}}{\ln (1 / \kappa)}\right)}\left[1-\left(\frac{r}{R}\right)^{2}-\frac{1-\kappa^{2}}{\ln (1 / \kappa)} \ln \left(\frac{R}{r}\right)\right]+\omega \frac{\ln (R / r)}{\ln (1 / k)}(9)
$$

In the motionless condition, the velocity profiles present a parabolic shape for $\mathrm{Re}_{h}=$ 40 and 105. However, radial transport of momentum yields to a flatter distribution for $\mathrm{Re}_{h}=213$. The experimental results for the counter-current velocity profiles present a similar behaviour, with an earlier onset to this velocity pattern, that plays a role on the appearance of low Reynolds number turbulent flow characeristics. For $\omega=-2$, a flat velocity distribution is found for Reynolds number $\operatorname{Re}_{h}=40$ and 80 . 


\subsection{Co-current mode}

During the co-current semi-cycle, three different flow patterns may appear, depending on the sign of the blockage parameter $\beta$. Experimental results for Reynolds number $\operatorname{Re}_{h}=80$ and velocity ratios $\omega=0.5,1$ and 2 are presented in Fig. 12 and discussed below.

- $\omega=0.5(\beta>0)$. In this working mode, the scraper moves in the same direction of the mean flow, but with lower velocity $v_{p}=v_{f} / 2$. Thus, the insert device imposes a blockage over the flow, that is forced to deviate when it encounters the moving plugs. The nature of the flow is therefore similar to that described for counter-current conditions, although some features do not exist for $\omega<1$. Actually, no reversed flow is found in the rear side of the plugs, indicating the absence of recirculation bubbles when the flow expands, due to the co-current movement of the scraper. Likewise, no transverse vortex appears in the front side of the plugs, as can be seen in detail in Fig. 13, for the tested velocity ratios. Nonetheless, transverse vortices have been reported in co-current mode, with the aid of numerical simulation, for very low velocity ratios $(\omega \leq 0.1)$ and high Reynolds numbers.

The blockage of the plugs increases the global velocity level. Velocity profiles in the control axial position are shown in Fig. 14 (left), for Reynolds numbers $\omega=0.5$ and $\operatorname{Re}_{h}=80,150$ and 250. A parabolic shape is reported in all the

conditions, with similar maximum velocity values, of the order of $v_{\text {max }} / v_{\text {med }} \approx$ 1.8. The absence of recirculation bubbles justifies the lower velocity values in this acceleration region. This is the reason of the similar velocity levels encountered for all the Reynolds numbers. It is also noticeable the fact that no evolution of the parabolic shape towards a flatter velocity profile is found for 
the higher Reynolds number tests. This could mean that the lower blockage of the plug in co-current conditions allows stabilizing laminar flow conditions for a wider working regime, rather than acting as a turbulence promoter.

- $\omega=1(\beta=0)$. The scraper and the mean flow move with the same velocity, thus minimizing the blockage imposed by the plugs. The flow is smoothly deviated in the vicinity of the plugs, but a similar pattern is observed upstream and downstream these elements. The axial velocity profile, depicted in Fig. 14 (center), presents a parabolic shape with a fair agreement with the analytical solution for the equivalent concentric annuli with co-current moving core. This tendency is preserved for all the Reynolds numbers under investigation $(40 \leq$ $\mathrm{Re}_{h} \leq 250$ ), indicating again that the flow keeps laminar for this working condition in a wider range of Reynolds numbers, with respect to the motionless characteristics.

- $\omega=2(\beta<0)$. The scraper moves faster than the mean flow, dragging it locally in the surroundings of the plugs. As a consequence, the flow presents a completely different pattern to those studied so far. The flow in the front and rear side of the plugs has the same direction of the mean flow. However, as the scraper velocity is higher than the flow velocity, $v_{p}=2 \times v_{f}$, the movement of the plugs not only drags the flow over their front and rear sides, but also generates a suction effect on the opposite tube section, yielding to a single recirculation bubble that extends backwards, with low local velocity levels of the order of $v / v_{\text {med }} \approx 1$. Velocity profiles are shown in Fig. 14 (right) for Reynolds number $\operatorname{Re}_{h}=40$ and 80, contrasted with the analytical solution for the equivalent concentric annuli. 


\section{Pressure drop results}

\subsection{Counter-current}

Fanning friction factor results in counter-current conditions present a twofold interest: in the frame of this work, the evolution of friction factor with Reynolds number and velocity ratio provides indirect information on the nature of the flow, allowing to identify pure laminar, transitional and turbulent flow. It also gives information on the influence of the working regime in maximum pressure drop. This is of primary importance, for example, in applications where a minimum mass flow must be continuously pumped despite the adverse conditions present during counter-current semi-cycles.

Fig. 15 presents Fanning friction factor results in counter-current conditions for velocity ratios $\omega=-0.5,-1$ and -2 and maximum values of Reynolds number of $\operatorname{Re}_{h} \approx 1000$. Results for the motionless scraper $(\omega=0)$ are also included as a reference, together with the analytic laminar solution for the equivalent smooth tube and the concentric annuli. The analytical solution of the latter with inner moving rod reads:

$$
f_{h}=\frac{16(1-\kappa)^{2}}{R e_{h}}\left[\frac{1-\omega\left(\frac{1}{2 \ln (1 / \kappa)}-\frac{\kappa^{2}}{1-\kappa^{2}}\right)}{\frac{1-\kappa^{4}}{1-\kappa^{2}}-\frac{1-\kappa^{2}}{\ln (1 / \kappa)}}\right]
$$

For $\omega=-0.5$, a laminar region is identified, where friction factor dependence on

Reynolds number is similar to motionless results, $f_{h, c c} \propto \mathrm{Re}_{h}{ }^{-0.75}$. However, the change of this tendency occurs at $\operatorname{Re}_{h} \approx 100$, which implies earlier transitional flow (the onset in motionless conditions is reported for $\mathrm{Re}_{h} \approx 200$ ). Pressure drop augmentations of $70 \%$ are found in laminar regime, referenced to $\omega=0$, and $150 \%$ in turbulent flow $\left(\operatorname{Re}_{h} \approx 700\right)$, as a result of the turbulence advance. 
A similar trend is found for $\omega=-1$ and $\omega=-2$. However, the increase of velocity ratio yields to a lower influence of Reynolds number in friction factor in the laminar regime, $f_{h, c c} \propto \mathrm{Re}_{h}{ }^{-0.66}$. The transition from laminar to turbulent flow can not be inferred from the slope change detected in the data. The intersection of laminar-side and turbulent-side trend lines allows estimating transitional regime at $\operatorname{Re}_{h} \approx 70$ for $\omega=-1$ and $\operatorname{Re}_{h} \approx 40$ for $\omega=-2$. These results confirm the effect of the scraping velocity on turbulence promotion in counter-current conditions.

Similarly, pressure drop increases for increasing velocity ratios. Maximum augmentations of $260 \%$ are found for $\omega=-2$ in laminar regime, whereas turbulent flow augmentations of $900 \%$ are can be inferred from the experimental data, with respect to the motionless conditions.

\subsection{Co-current}

Experimental results of Fanning friction factor are represented in Fig. 16, for $\omega=0,0.5$ and 1 . The co-current movement of the scraper decreases the pressure drop across the tube for all the conditions with respect to the motionless scraper, as a result of the lower blockage imposed by the moving plugs, and the decreased shear rate resulting in the flow.

The data trend for $\omega=0.5$, covering the range $15<\operatorname{Re}_{h}<300$, presents the characteristic behaviour of laminar flow, with $f_{h, e q} \propto \mathrm{Re}_{h}{ }^{-0.85}$. Unlike the results for $\omega=0$, no onset of transition is observed for the Reynolds number range tested, following that the flow pattern induced by the co-current movement of the scraper delays the appearance of turbulence phenomena. This conclusion fully agrees with the velocity profiles of Fig. 14, that keep a parabolic shape even for $\mathrm{Re}_{h}=250$.

The results for $\omega=1$ are also included in Fig. 16, together with the solution of Fanning friction factor for the concentric annuli with co-current moving core and 
$\omega=1$. The fair agreement between the experimental solution for moving scraper and the analytical solution for concentric annuli reveals the negligible blockage of the scraper when moving with same velocity and direction than the mean flow. This fact is also proved by the similarity of the experimental and analytical velocity profiles for this velocity ratio.

The performance of the scraper for $\omega=2$ presents a different pattern, owing to the negative blockage imposed by the scraper, that moves faster than the fluid. Actually, in these conditions the scraper adds mechanical energy to the flow, working as a pump inside the tube. As a result, a pressure increase is measured across the test section for $\omega=2$, yielding subsequent negative friction factors. The accuracy of the piezorresistive pressure sensors prevents from obtaining reliable results for the magnitudes measured in this working condition, that finally retrieve spurious friction factor values. The numerical simulation will be employed next to overcome this inconvenience and provide a physical insight into this phenomenon.

\section{Numerical wall shear profiles}

Numerical simulations of the flow were performed in the range of Reynolds number between 15 and 170, under the assumption of laminar flow. Solutions for countercurrent and co-current motion were obtained with velocity ratios $\omega= \pm 0.5, \pm 1$ and \pm 2 . Velocity profiles in the radial section located at $P / 5$ of the front side of the plugs are extracted for $\mathrm{Re}_{h}=80$ for counter-current and co-current motion. The comparison with experimental results is represented in Fig. 17, showing a satisfactory agreement. Higher discrepancies are found in the vicinity of the tube wall and rod, as a result of the experimental restrictions to properly iluminate those regions of the insert tube. On the contrary, the numerical simulation successfully retrieves the slip condition in the scraper rod, $r / R=\kappa$, for all the operating conditions. 
In counter-current motion, maximum values of the skin friction coefficient are found in the front side of the plugs, towards the part of the tube where the bulk flow is deviated (see Fig. 18). The region of high shear stress extends towards the reduced cross-section region and further downstream. Progressively, the boundary layer grows along the tube wall side opposite to the plugs, yielding to a reduction of the local shear stress. Conversely, a huge region of low wall shear stress is found in the rear side of the plugs. This area is influenced by the lower local velocity of the recirculation bubbles generated when the flow expands downstream the plugs. This distribution is basically repeated for the three velocity ratios.

In co-current motion, the existence of three different flow patterns implies different shearing profiles, which are presented in Fig. 19. For $\omega=0.5$, the skin friction coefficient distribution follows a similar pattern to the one obtained above for positive blockage situations presented above: lower shear stress are found downstream the plugs, although there are no recirculation bubbles owing to the parallel motion of the scraper and fluid. However, the expansion of the flow that overcomes the plugs yields to lower velocities in this area. The acceleration of the flow and further deviation generates a high local shear stress field as well. For $\omega=1$, only higher distributions are found in the deviation of the flow opposite to the plugs. The region in-between presents a fairly constant shear field, owing to the slight perturbance of the flow. For $\omega=2$, the higher shear stresses are found upstream and downstream the plugs, owing to the acceleration of the flow forced by the scraper movement. Conversely, lower shear stress are found on the opposite part of the tube where the plugs are located, due to the recirculation bubble created by the dragged flow.

The influence of pressure and viscous forces on the global pressure drop can also be accounted for with the aid of numerical simulation. Both effects have been analyzed integrating the viscous forces, which are dominant in the tube and rod walls, and 
pressure forces, that mainly appear on the plug surfaces normal to the flow direction. To account for this analysis, a periodic control volume extended over the scraper pitch is chosen. Two plugs are contained in this volume, thus contributing with net pressure forces over their rear and front faces. Momentum conservation over the pitch allows establishing the next forces relation:

$$
\underbrace{\int_{\sigma_{1}}^{\sigma_{2}} p \vec{n} d \sigma}_{\text {totalforce }}=\underbrace{\int_{\text {wall }} \overline{\bar{\tau}} \vec{n} d \sigma+\int_{\text {rod }} \overline{\bar{\tau}} \vec{n} d \sigma}_{\text {viscousforce }}+\underbrace{\int_{\text {plugs }} p \vec{n} d \sigma}_{\text {pressureforce }}
$$

where $\sigma_{1}$ and $\sigma_{2}$ are the annular cross-sections of the tube limiting the domain.

Fig. 20 presents the pressure, viscous and total force coefficients over a scraper pitch, obtained for the numerical simulations of counter-current motion. Pressure forces exceed the viscous contribution in all working conditions. For $\omega=-0.5$, these forces are of the same order for the lowest Reynolds numbers $\left(\operatorname{Re}_{h} \approx 15\right)$, whereas for $\operatorname{Re}_{h} \approx 150$, the contribution of pressure forces to total force is of the order of 80\%. Experimental results of Fanning friction factor are contrasted with total force coefficient by means of

$$
c_{F}=f_{h} \frac{4 P}{D_{h}}
$$

Excellent agreement of experimental data with total force numerical computation is found for $\omega=-0.5$. For $\omega=-1$, the same tendency is observed, with a contribution of pressure forces of $85 \%$ at $\operatorname{Re}_{h} \approx 150$. For $\omega=-2$, pressure forces play a fundamental role even for the lowest Reynolds numbers, highlighting the negative effect of the scrapers motion in terms of loss of mechanical energy. In this working condition, pressure forces contribution is as high as $70 \%$ for $\operatorname{Re}_{h} \approx 15$ and $90 \%$ fo $\operatorname{Re}_{h} \approx 150$. Agreement of numerical results with experimental data fails for $\operatorname{Re}_{h}>80$, which may indicate the existence of a different flow nature, with turbulent and unsteady 
characteristics. The numerical study of these flow features is beyond the scope of this work.

Numerical results in co-current motion are presented in Fig. 21. Viscous force for $\omega=0.5$ is dominant for the range of Reynolds number under investigation. For $\operatorname{Re}_{h} \approx 150$, viscous and pressure forces are of the same order, and the data trend allows inferring that, for higher Reynolds numbers, pressure forces would exceed viscous effects. Agreement with experimental results derived from Fanning friction factor is excellent.

For $\omega=1$, pressure forces are negligible for the whole range of Reynolds numbers. This result is expected from the fact that the scraper and the fluid move with similar velocity, thus minimizing the blockage imposed by the plugs. Total pressure drop in this working condition is therefore caused exclusively by viscous effects.

For $\omega=2$, a completely different behaviour is found: pressure force coefficient is negative, as a result of the faster velocity of the scraper within the flow. Higher pressure fields are reported in the rear faces of the plugs, thus resulting in net pressure forces opposite to the mean flow direction. For this velocity ratio, this pressure contribution yields to negative total force coefficients. Contrast with experimental data are also reported. A reasonable agreement is found, as expected from the negative Fanning friction coefficients obtained in the test bench. As discussed above, sensors accuracy prevented from obtaining more reliable experimental data for $\omega=2$.

\section{Conclusions}

1. Flow pattern and shearing profiles investigation is carried out in tubes of heat exchangers with reciprocating scrapers. PIV measurements are complemented with pressure drop tests and CFD computations, at low Reynolds numbers 
and velocity ratios $\omega=0, \pm 0.5, \pm 1$ and \pm 2 , in order to obtain a global understanding of the flow behaviour.

2. Flow structures in counter-current motion are similar to those found for the motionless condition, owing to the positive blockage imposed by the scraper. Velocity ratio plays an important role on the radial transport of momentum, yielding to the promotion of turbulent flow characteristics.

3. Flow behaviour in co-current mode presents three different patterns depending on the blockage parameter: the flow may present a meandering path for positive blockage, no disturbances for zero-blockage, and a dragging pattern for negative blockage.

4. Fanning friction factor during both scraping semi-cycles have been experimentally obtained, for different velocity ratios. Pressure drop augmentations in counter-current mode have been assessed, as well as earlier transition to turbulence. Co-current motion of the scraper have proved to preserve laminar flow for a wider range of Reynolds number.

5. Numerical simulation of the flow provides an insight into the wall shear stress, and allows identifying the contribution of the local flow structures to the pressure losses mechanism. The higher contribution of drag losses to global pressure drop has been assessed for counter-current conditions. In co-current mode, the influence of the blockage on head losses is determined, and the drag effect for $\omega=2$ is clearly defined. 


\section{Acknowledgements}

This research has been partially financed by the DPI2007-66551-C02 grant of the Spanish Ministery of Science and the company "HRS Spiratube". The authors are grateful to SEDIC-SAIT (UPCT) for providing the technical resources for CFD computations.

\section{References}

[1] C. S. Rao, R. W. Hartel, Scraped Surface Heat Exchangers, Critical Reviews in Food Science and Nutrition 46 (2006) 207-219.

[2] A. E. Bergles, S. D. Joshi, Augmentation techniques for low Reynolds number in-tube flow, Low Reynolds Number Flow Heat Exchangers, Hemisphere, Washington D.C., 1985.

[3] A. M. Trommelen, W. J. Beek, Flow phenomena in a scraped surface heat exchanger (Votator-type), Chemical Engineering Science 26 (1971) 1933-1942.

[4] M. Stranzinger, A. Bieder, K. Feigl, E. Windhab, Effects of flow incidence and secondary mass flow rate on flow structuring contributions in scraped surface heat exchangers, Journal of Food Process Engineering 25 (2002) 159-187.

[5] E. Dumont, F. Fayolle, V. Sobolik, J. Legrand, Wall shear rate in the TaylorCouette-Poiseuille flow at low axial Reynolds number, International Journal of Heat and Mass Transfer 45 (2002) 679-689.

[6] M. Harrod, Scraped surface heat exchangers: A literature survey of flow patterns, mixing effects, residence time distribution, heat transfer, and power requirements, Journal of Food Process Engineering 9 (1986) 1-62. 
[7] A. García, J. P. Solano, P. G. Vicente, A. Viedma, Flow pattern assessment in tubes with wire coil inserts in laminar and transition regimes, International Journal of Heat and Fluid Flow 28 (2007) 516-525

[8] M. Raffel, C. Willer,J. Kompenhans, Particle Image Velocimetry: A practical guide, first ed., Springer, 2000.

[9] F. Scarano, M. L. Reithmuller, Advances in iterative multigrid PIV image processing, Experiments in Fluids 29 (2000) 51-60.

[10] ISO, Guide to the expression of uncertainty in measurement, first ed., ISBN 9267-10-188-9, International Organization for Standarization, Switzerland, 1995.

[11] J. Y. Luo, R. I. Issa, A. D. Gosman, Prediction of Impeller-Induced Flows in Mixing Vessels Using Multiple Frames of Reference, IChemE Symposium Series 136 (1994) 549-556.

[12] I. B. Celik, U. Ghia, P. J. Roache, C. J. Freitas, H. Coleman, P. E. Raad, Procedure for Estimation and Reporting of Uncertainty Due to Discretization in CFD Applications, Journal of Fluids Engineering 130 (2008) 078001-1-4. 


\section{Figure captions}

Figure 1: Detail of the scraper geometry inserted in the tube. Typical designs cover the total length of the tube for full cleaning

Figure 2: Experimental setup for PIV: (1) reservoir tank and filter, (2) honeycomb, (3) test section, (4) hydraulic piston, (5) reservoir tank, (6) electric heater, (7) electromagnetic flowmeter, (8) frequency converter, (9) centrifugal pump.

Figure 3: Scraper velocity for a characteristic scraping cycle

Figure 4: Optical sensor arrangement for phase-averaged PIV measurement in counter-current and co-current motion

Figure 5: Experimental set-up for pressure drop measurements: (1) reservoir tank, (2) positivedisplacement pump, (3) frequency converter, (4) control valve, (5) electrical heater, (6) Coriolis flowmeter, (7) oval-wheel flowmeter, $(8,9)$ inlet and outlet RTD probes, (10) differential pressure transducer, (11) hydraulic piston, (13) plate heat exchanger, (15) diverter valve, (16) PID controller, (17) cooling water, (19) cooling machine, $(12,14,18)$ centrifugal pumps.

Figure 6: Pressure drop signal across the test section for a characteristic scraping cycle: $\operatorname{Re}_{h}=60$, $\omega=0.1$

Figure 7: Detail of the structured mesh. Left: cross-sectional area. Right: Symmetry plane.

Figure 8: Assessment of mean flow structures measured in the symmetry plane (PIV)

Figure 9: PIV velocity field along the tube symmetry plane in counter-current motion, for $\operatorname{Re}_{h}=80$ and $\omega=-0.5,-1$ and -2 
Figure 10: Experimental results of the transverse vortex $\left(\vec{v} / v_{m e d}\right)$ for $\operatorname{Re}_{h}=80$ and $-2 \leq \omega \leq 0$

Figure 11: PIV velocity profiles for $\omega=0,-0.5,-1$ and -2 and several velocity ratios.

Figure 12: PIV velocity field along the tube symmetry plane in counter-current motion, for $\operatorname{Re}_{h}=$ 80 and $\omega=0.5,1$ and 2

Figure 13: Experimental results of the velocity field in the front side of the plug, for $\operatorname{Re}_{h}=80$ and $0 \leq \omega \leq 2$

Figure 14: PIV velocity profiles for $\omega=0.5,1$ and 2 and several velocity ratios.

Figure 15: Experimental results of Fanning friction in counter-current mode, for $\omega=-0.5,-1$ and -2 . Comparison with motionless results $(\omega=0)$ and analytical solutions for smooth tube and concentric annuli

Figure 16: Experimental results of Fanning friction in co-current mode, for $\omega=0.5$ and 1. Comparison with motionless results $(\omega=0)$ and analytical solutions for smooth tube, motionless concentric annuli and concentric annuli with internal co-current moving core $(\omega=1)$

Figure 17: Numerical velocity profiles for $\operatorname{Re}_{h}=80$ and several velocity ratios, in co-current and counter-current modes. Comparison with experimental data

Figure 18: Skin friction coefficient in the tube wall in counter-current motion, for $\operatorname{Re}_{h}=80$ and $\omega=-0.5,-1$ and -2

Figure 19: Skin friction coefficient in the tube wall in counter-current motion, for $\operatorname{Re}_{h}=80$ and $\omega=0.5,1$ and 2

Figure 20: Numerical computation of pressure, viscous and total force coefficients over a scraper pitch, in counter-current motion. Comparison with experimental data from pressure drop tests.

Figure 21: Numerical computation of pressure, viscous and total force coefficients over a scraper pitch, in co-current motion. Comparison with experimental data from pressure drop tests. 


\begin{tabular}{ccccccc}
\hline$\omega$ & -2 & -1 & -0.5 & 0.5 & 1 & 2 \\
\hline$v$ & 3.49 & 3.62 & 2.75 & 2.12 & 2.93 & 3.15 \\
$p$ & 0.23 & 0.85 & 0.78 & 0.69 & 0.53 & 0.55 \\
$c_{F}$ & 2.47 & 1.97 & 1.90 & 1.83 & 1.75 & 2.42 \\
\hline
\end{tabular}

Table 1: Grid Convergence Index (\%) for $\mathrm{Re}_{h}=80$ and different velocity ratios 


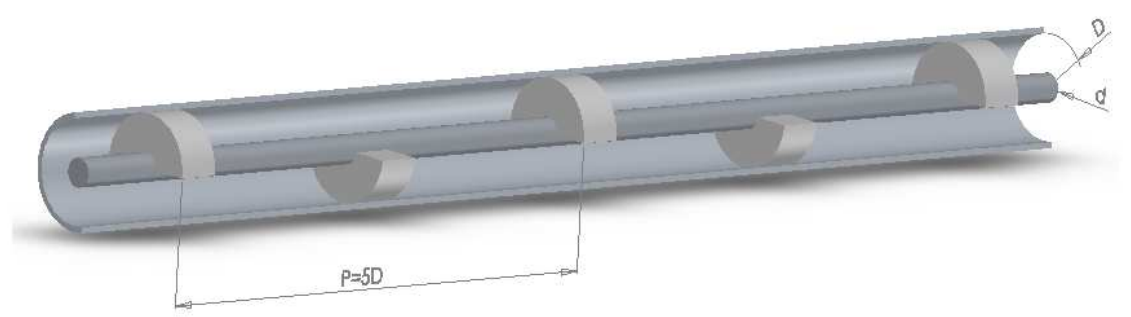




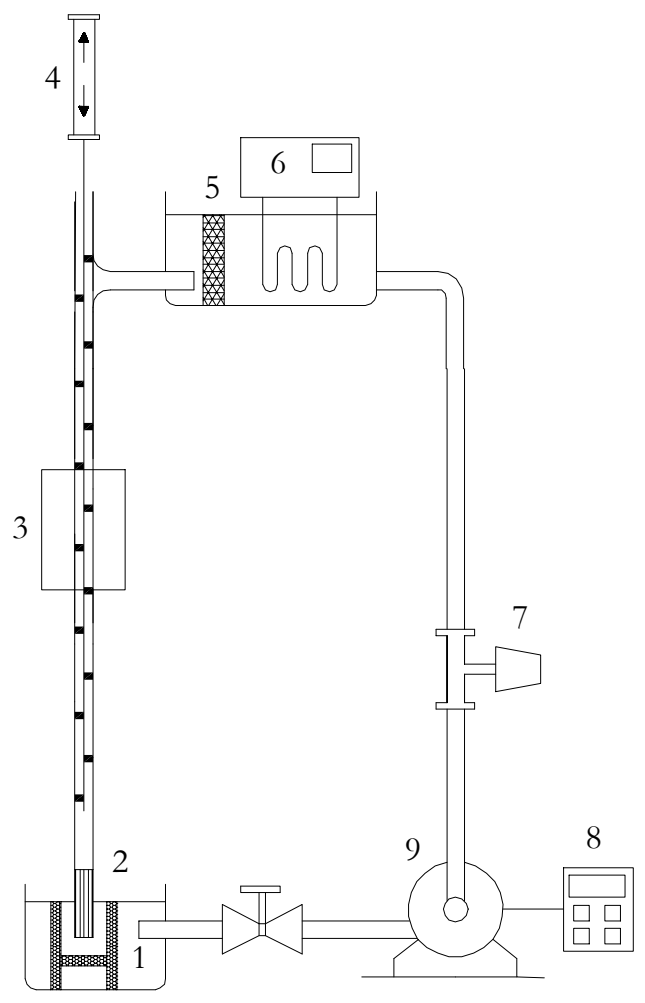




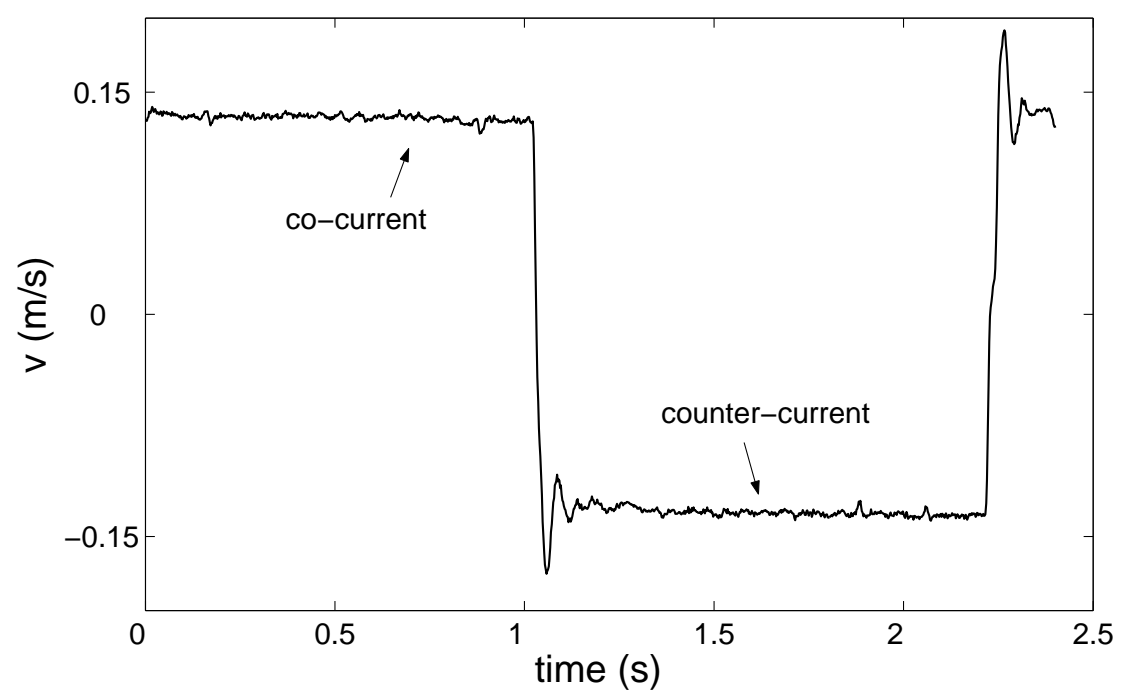



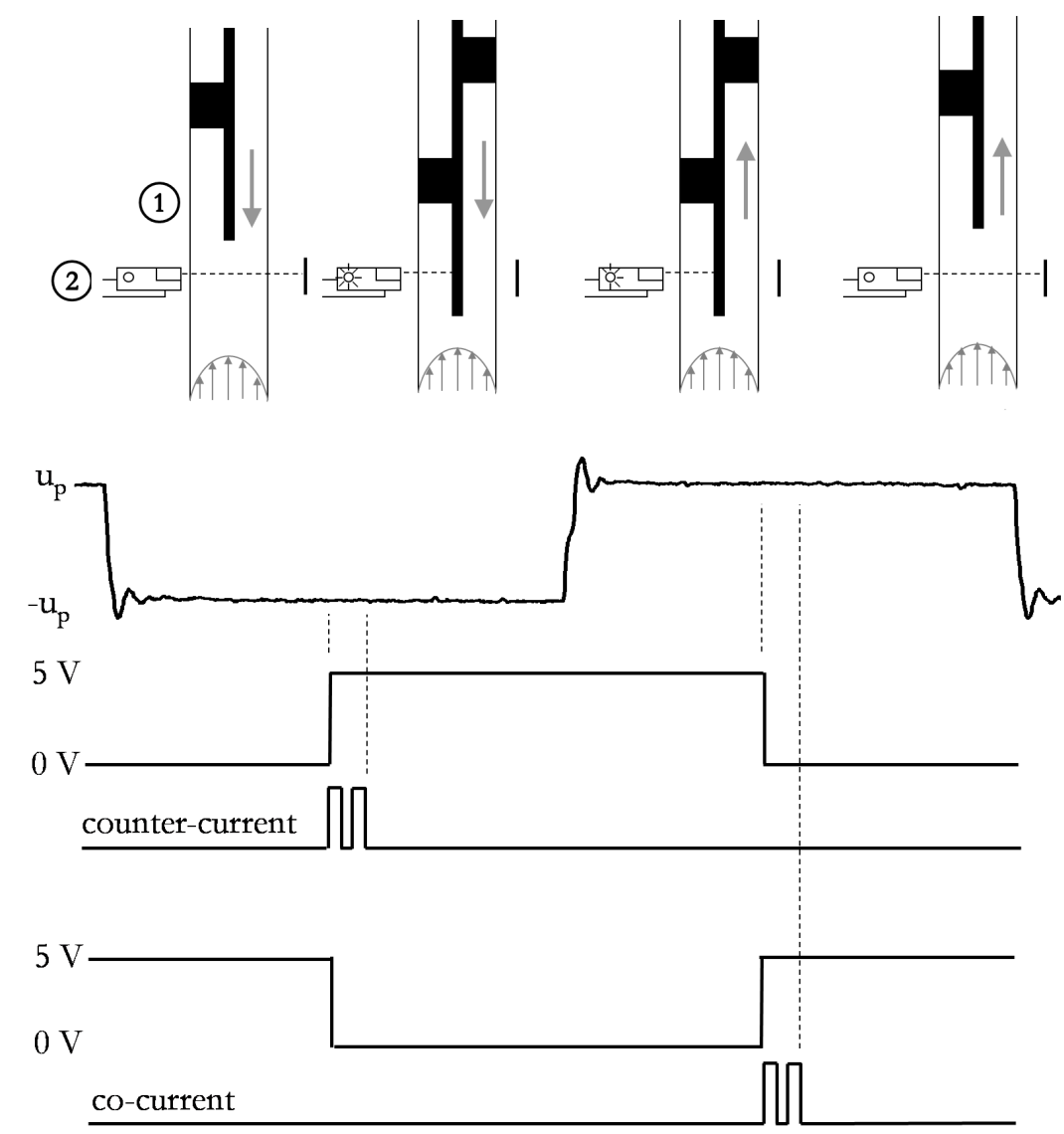


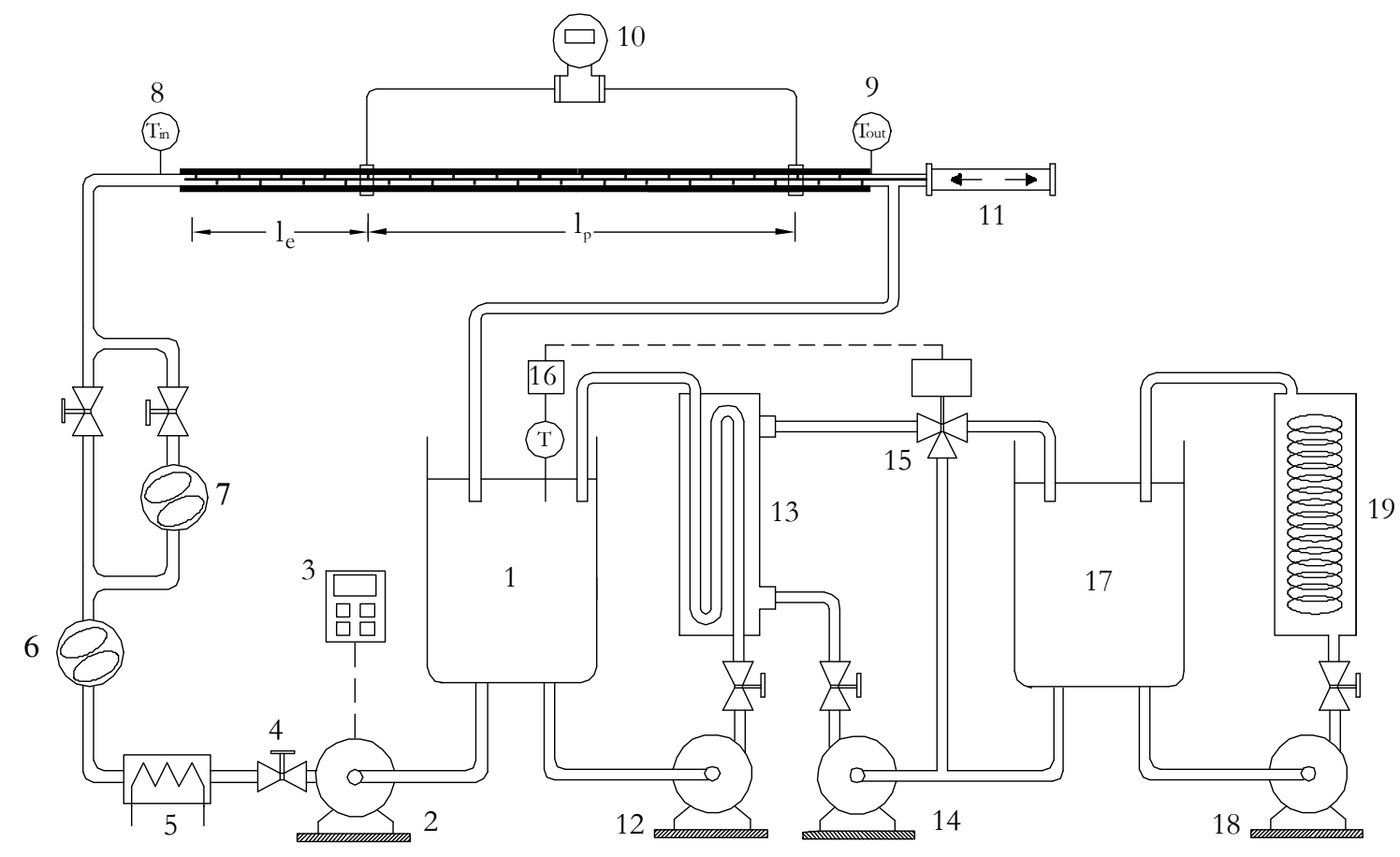




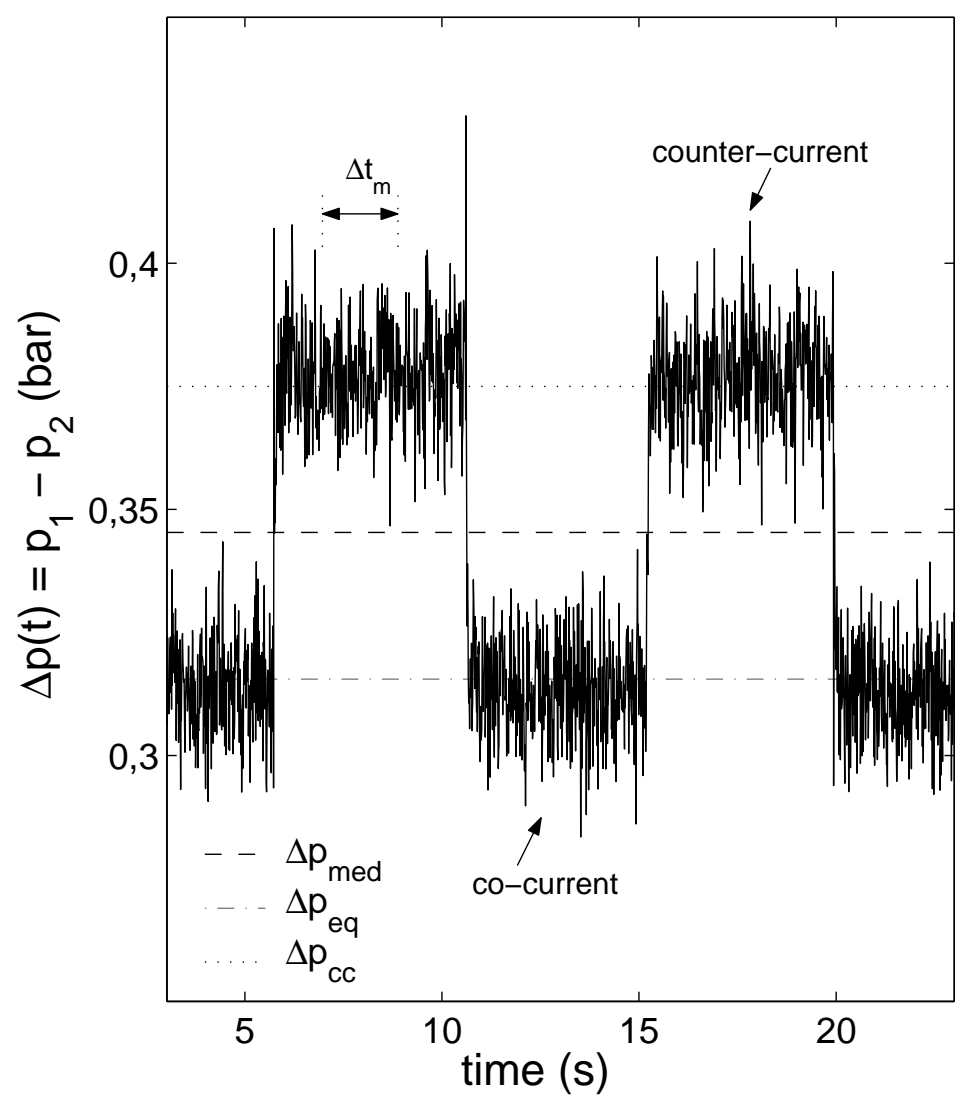



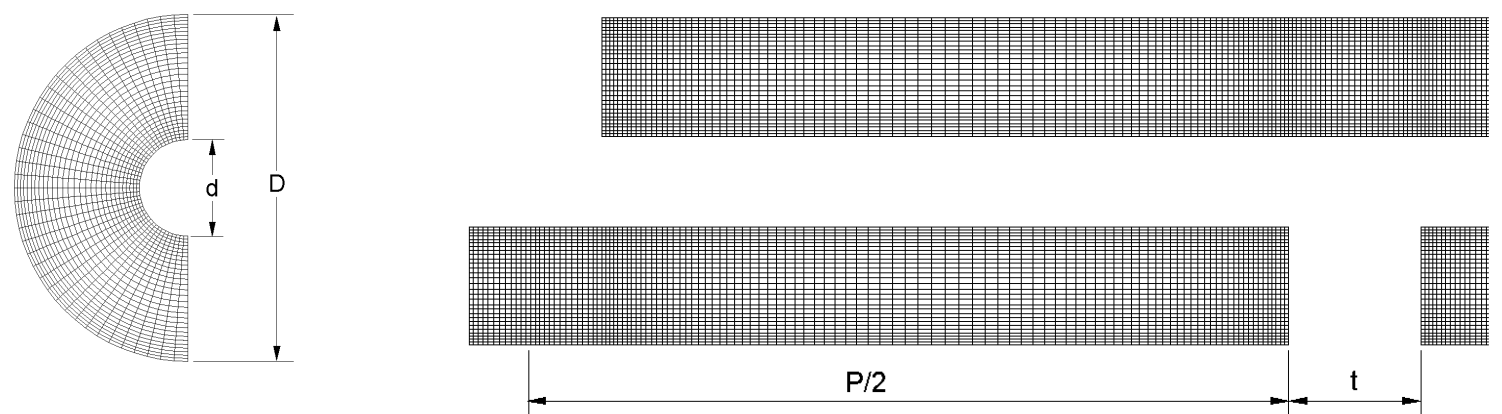


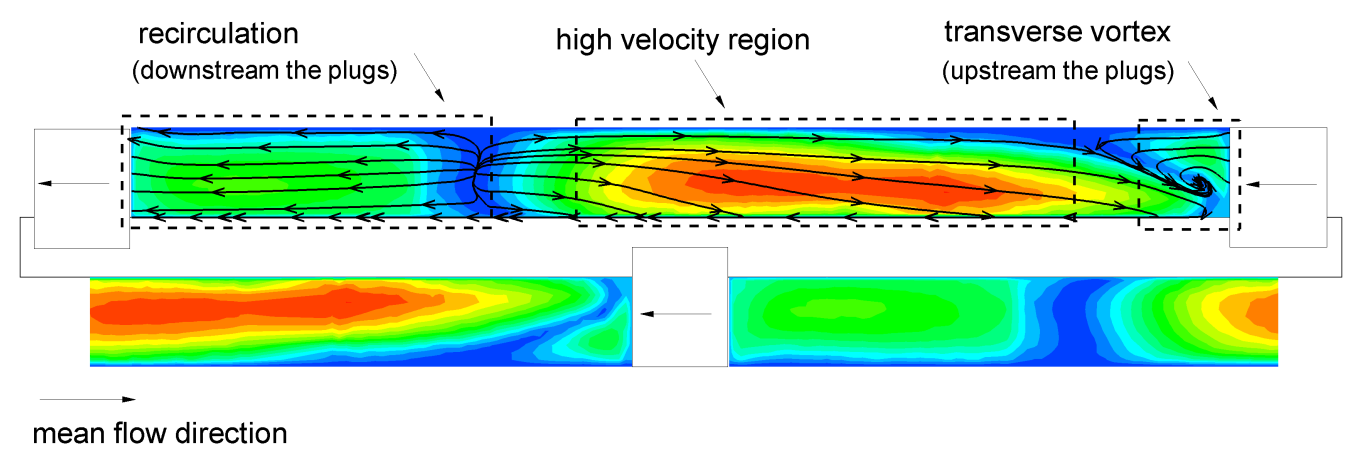




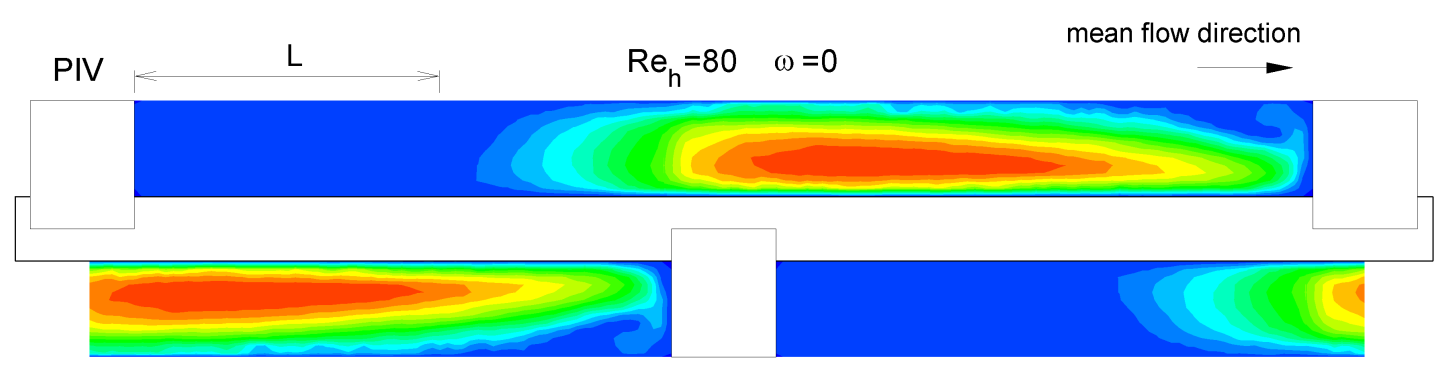

$\mathrm{v} / \mathrm{V}_{\text {med }}$

$\begin{array}{llllllllllllllll}0 & 0.2 & 0.4 & 0.6 & 0.9 & 1.1 & 1.3 & 1.5 & 1.7 & 1.9 & 2.1 & 2.3 & 2.6 & 2.8 & 3.0 & 3.2\end{array}$

$\mathrm{L} \quad \mathrm{Re}_{\mathrm{h}}=80 \quad \omega=-0.5$

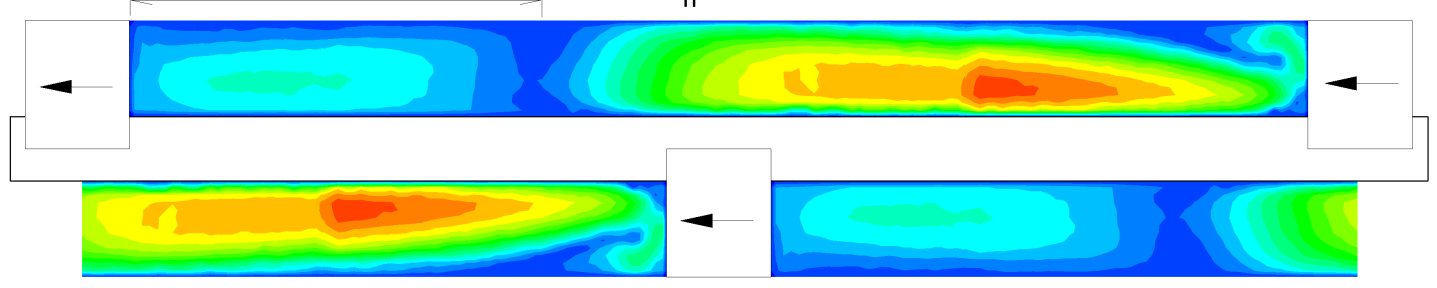

$\mathrm{v} / \mathrm{v}_{\mathrm{med}}$

$\begin{array}{llllllllllllllll}0 & 0.3 & 0.5 & 0.8 & 1.1 & 1.3 & 1.6 & 1.9 & 2.1 & 2.4 & 2.7 & 2.9 & 3.2 & 3.5 & 3.7 & 4.0\end{array}$

$\mathrm{L} \quad \mathrm{Re}_{\mathrm{h}}=80 \quad \omega=-1$

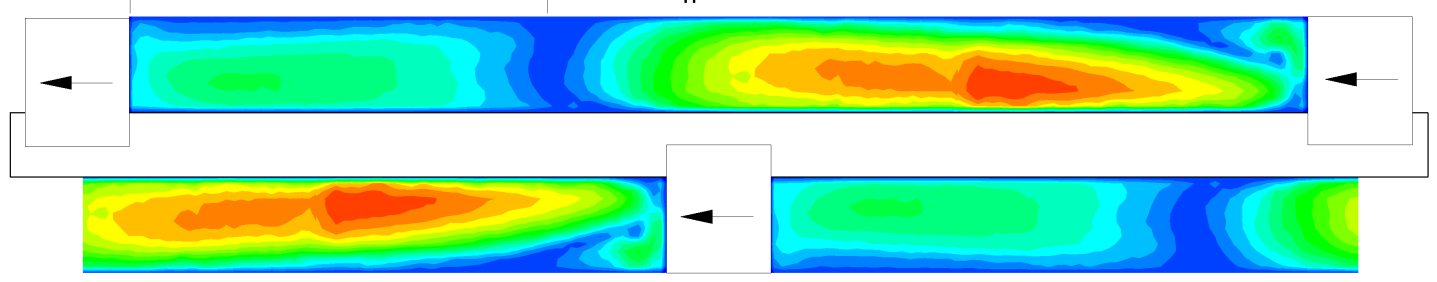

$\mathrm{v} / \mathrm{v}_{\text {med }}$

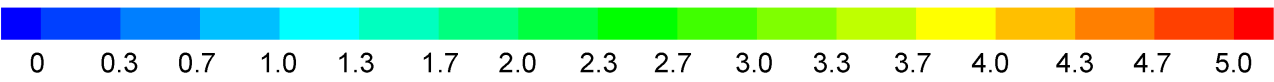

$\mathrm{L} \quad \Rightarrow \quad \mathrm{Re}_{\mathrm{h}}=80 \quad \omega=-2$

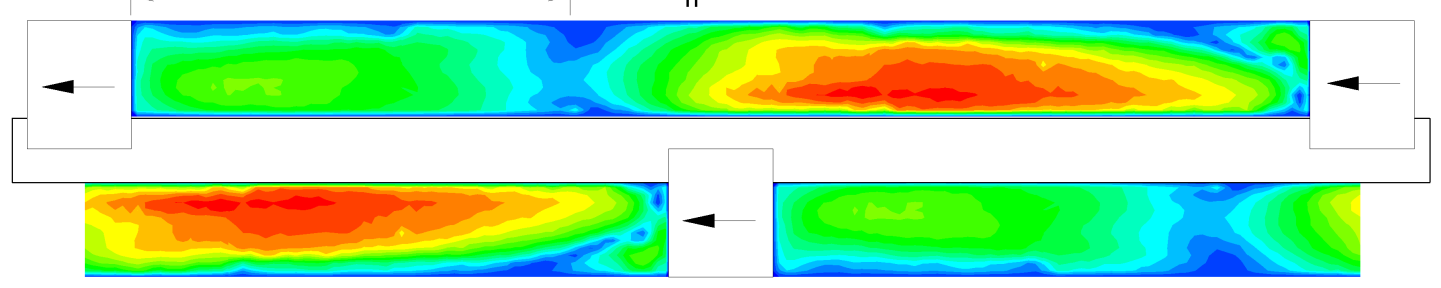

$\mathrm{v} / \mathrm{v}_{\mathrm{med}}$

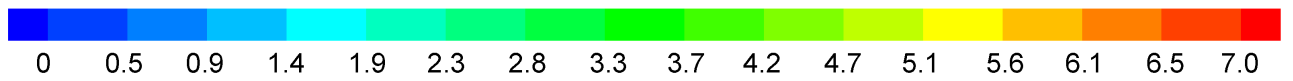



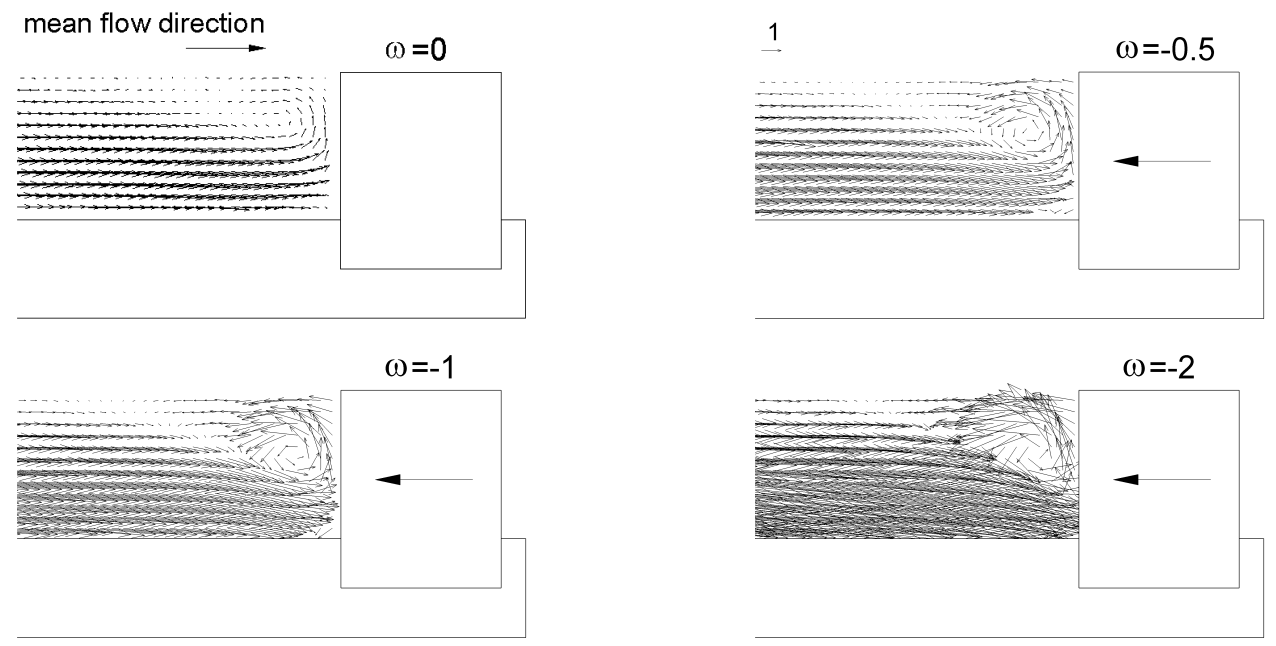

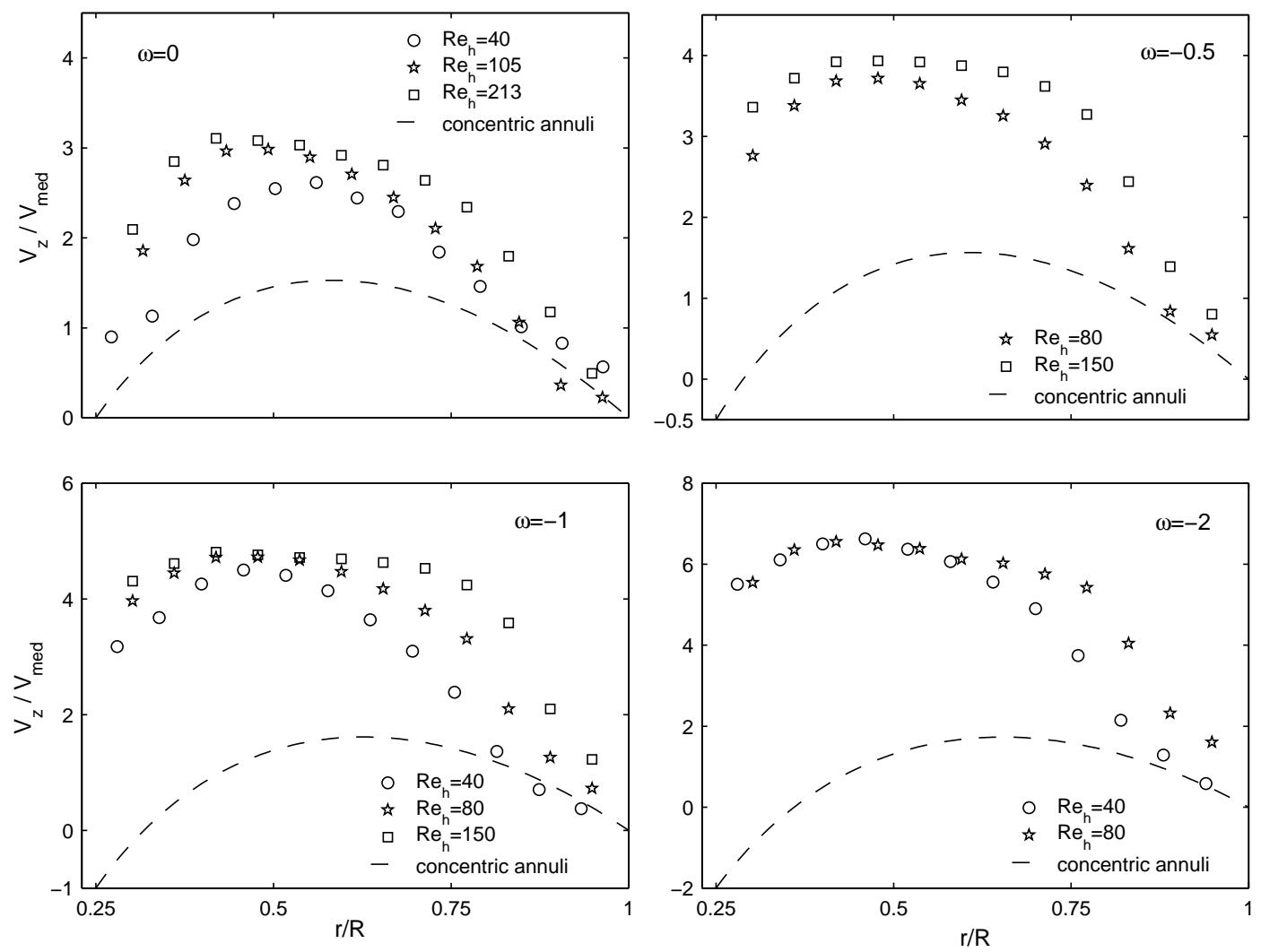


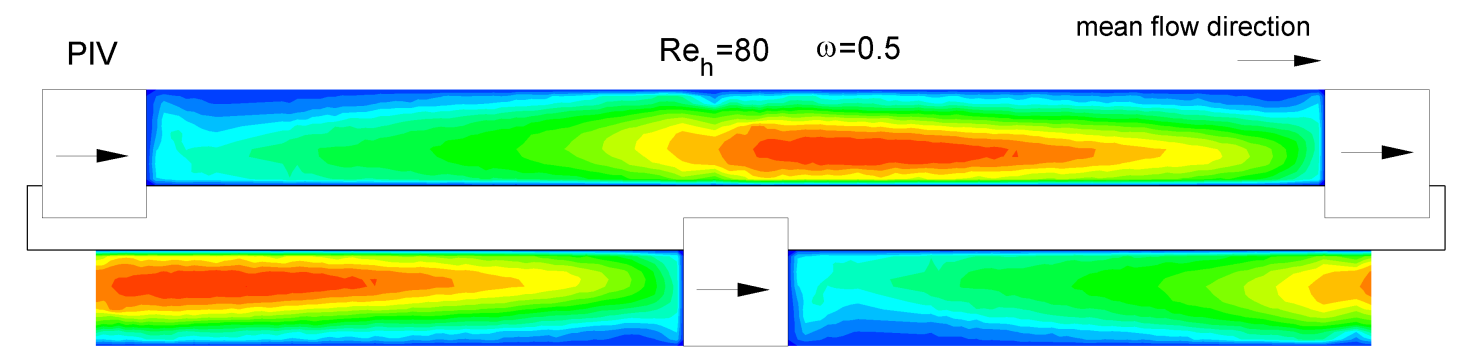

$\mathrm{v} / \mathrm{v}_{\mathrm{med}}$

$\begin{array}{llllllllllllllll}0 & 0.1 & 0.3 & 0.4 & 0.5 & 0.7 & 0.8 & 1.0 & 1.1 & 1.2 & 1.4 & 1.5 & 1.6 & 1.8 & 1.9 & 2.0\end{array}$

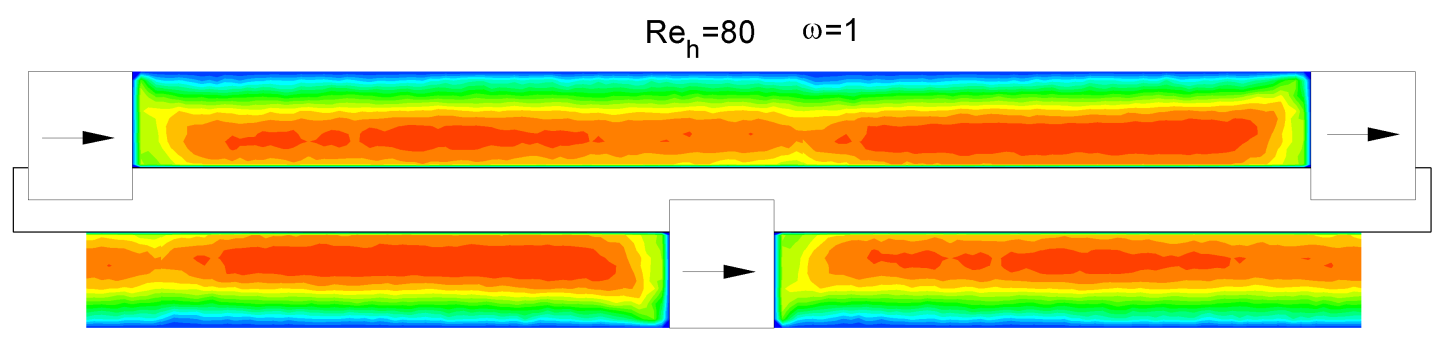

$\mathrm{v} / \mathrm{V}_{\mathrm{med}}$

$\begin{array}{llllllllllllllll}0 & 0.1 & 0.2 & 0.3 & 0.4 & 0.5 & 0.6 & 0.7 & 0.8 & 0.9 & 1.0 & 1.1 & 1.2 & 1.3 & 1.5 & 1.6\end{array}$

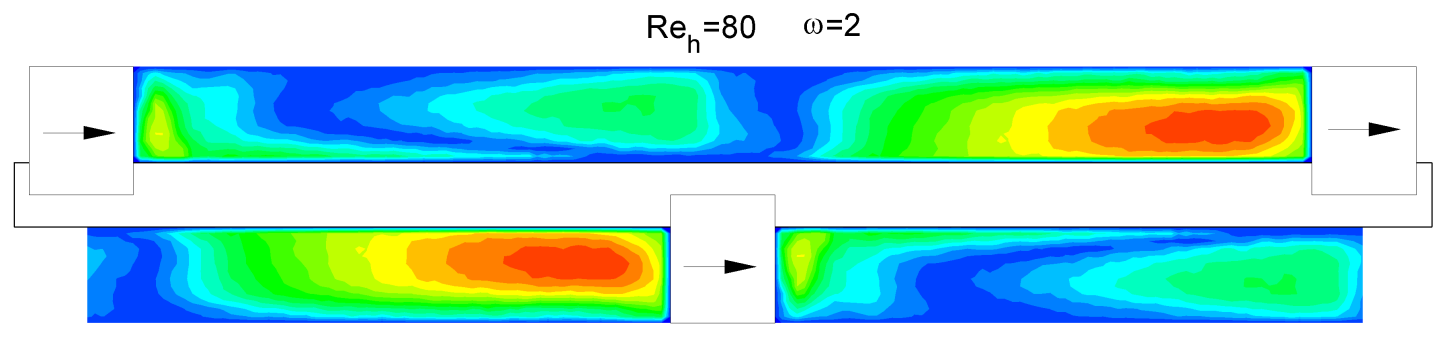

$\mathrm{v} / \mathrm{v}_{\mathrm{med}}$

$\begin{array}{llllllllllllllll}0 & 0.2 & 0.4 & 0.7 & 0.9 & 1.1 & 1.3 & 1.5 & 1.7 & 2.0 & 2.2 & 2.4 & 2.6 & 2.8 & 3.0 & 3.3\end{array}$ 

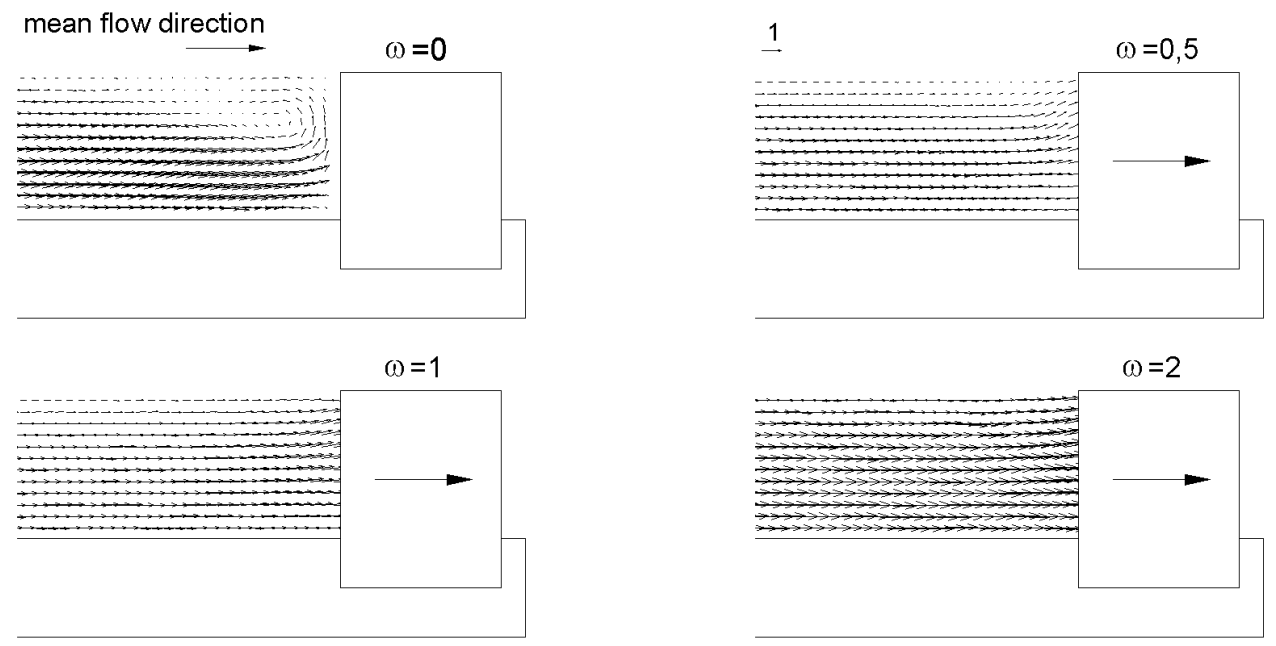

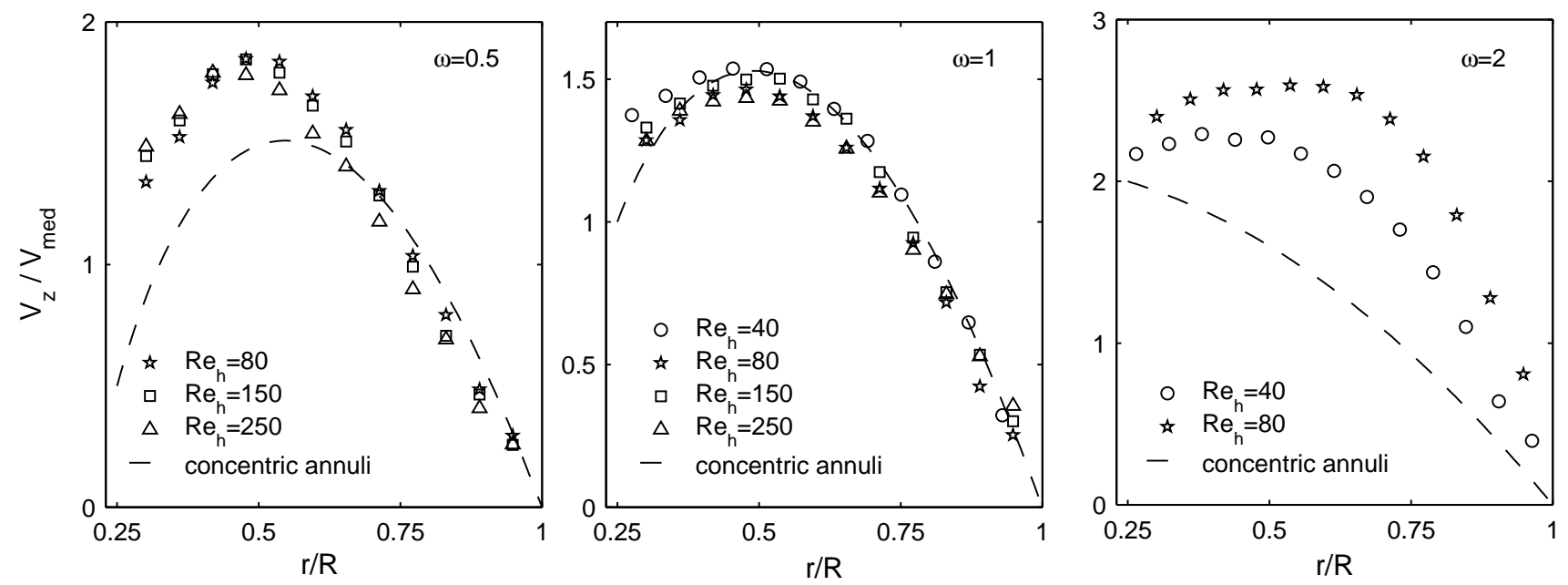


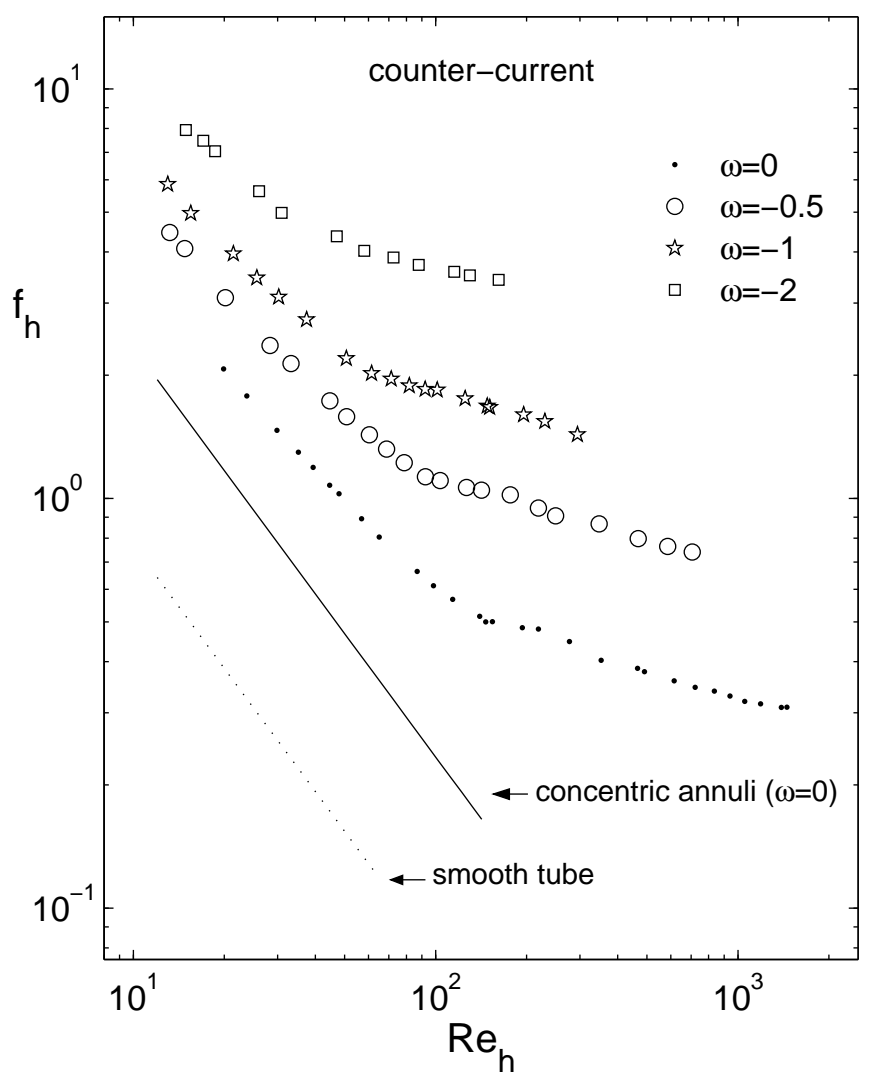




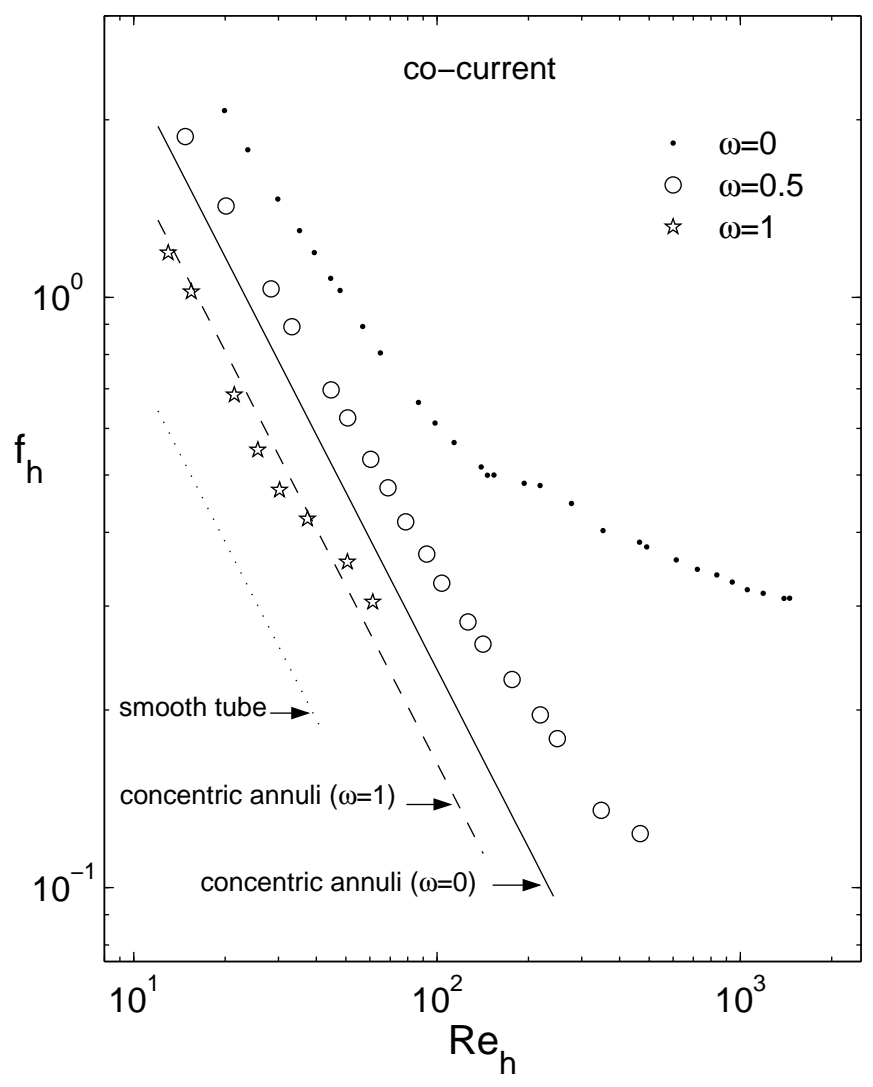



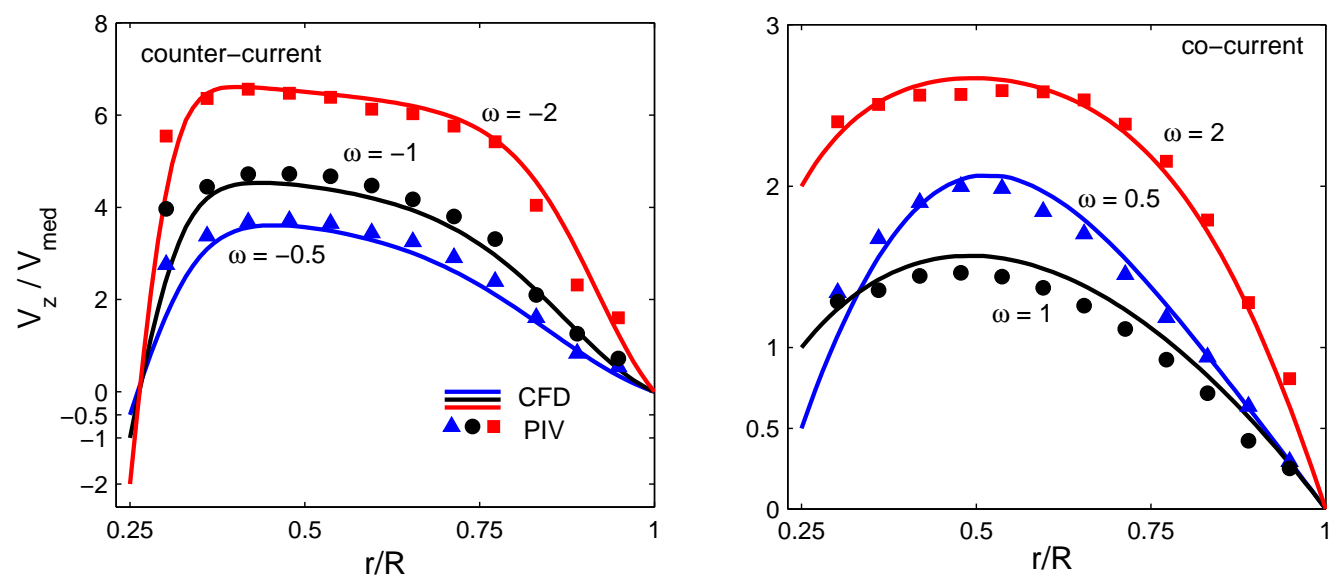

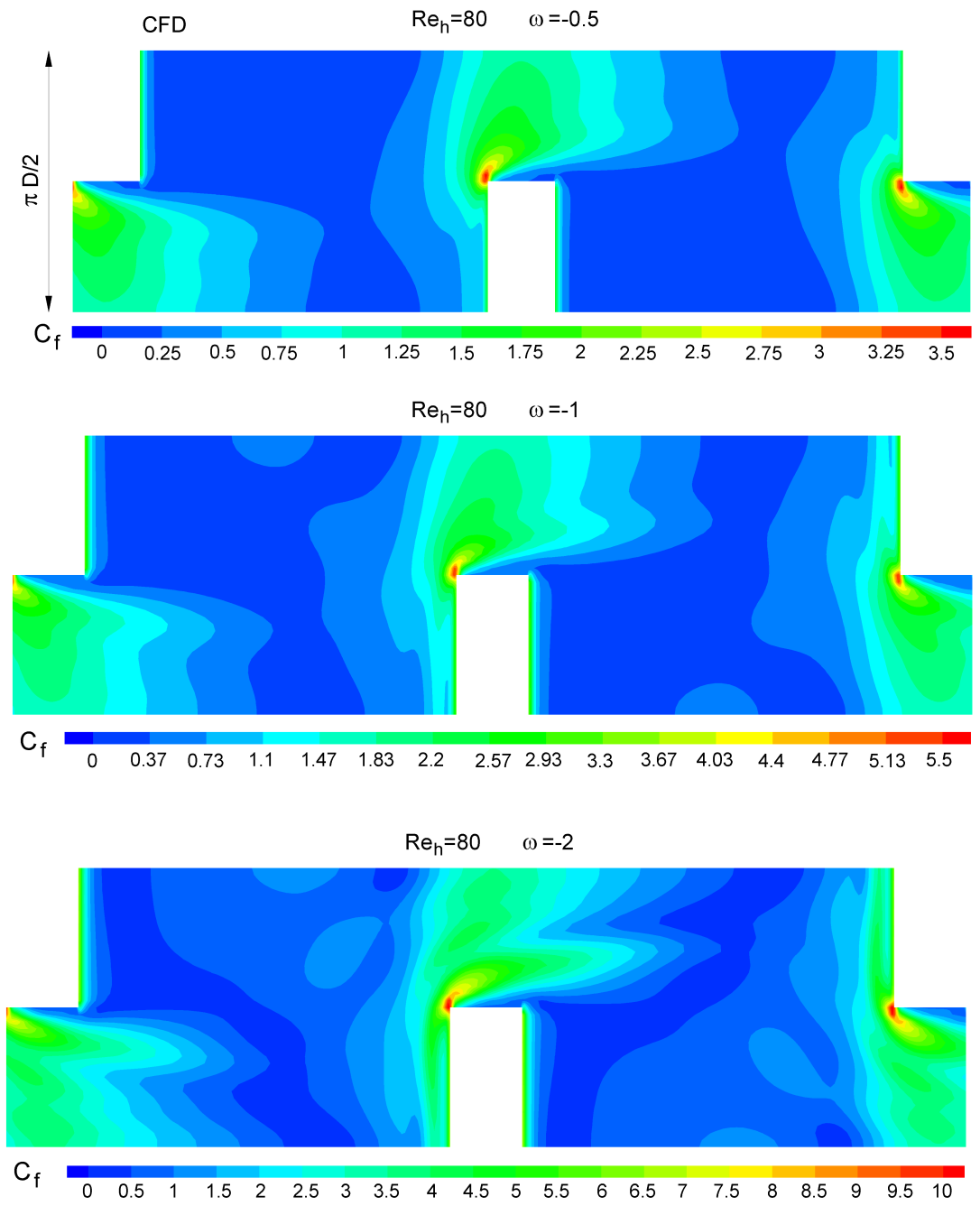

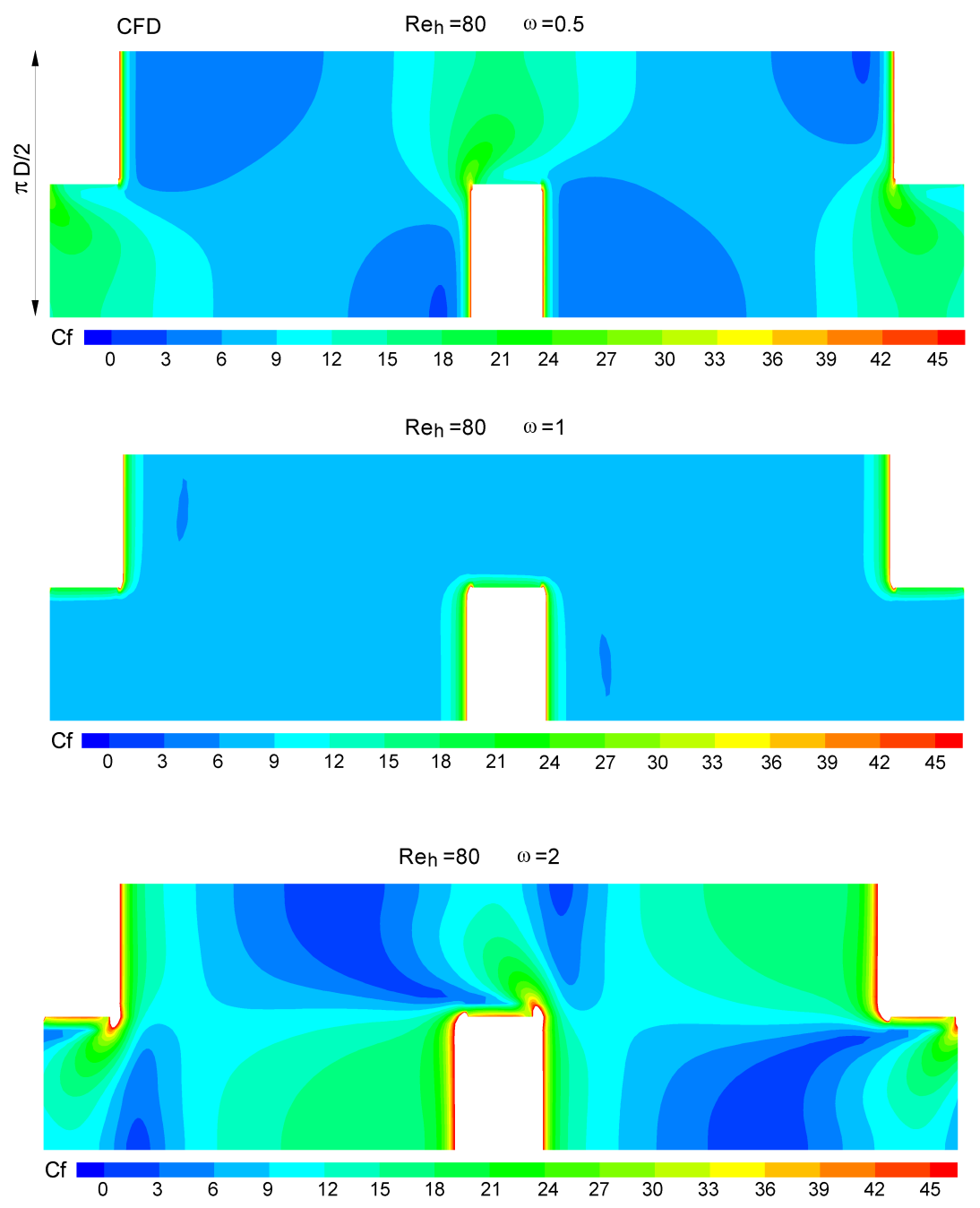


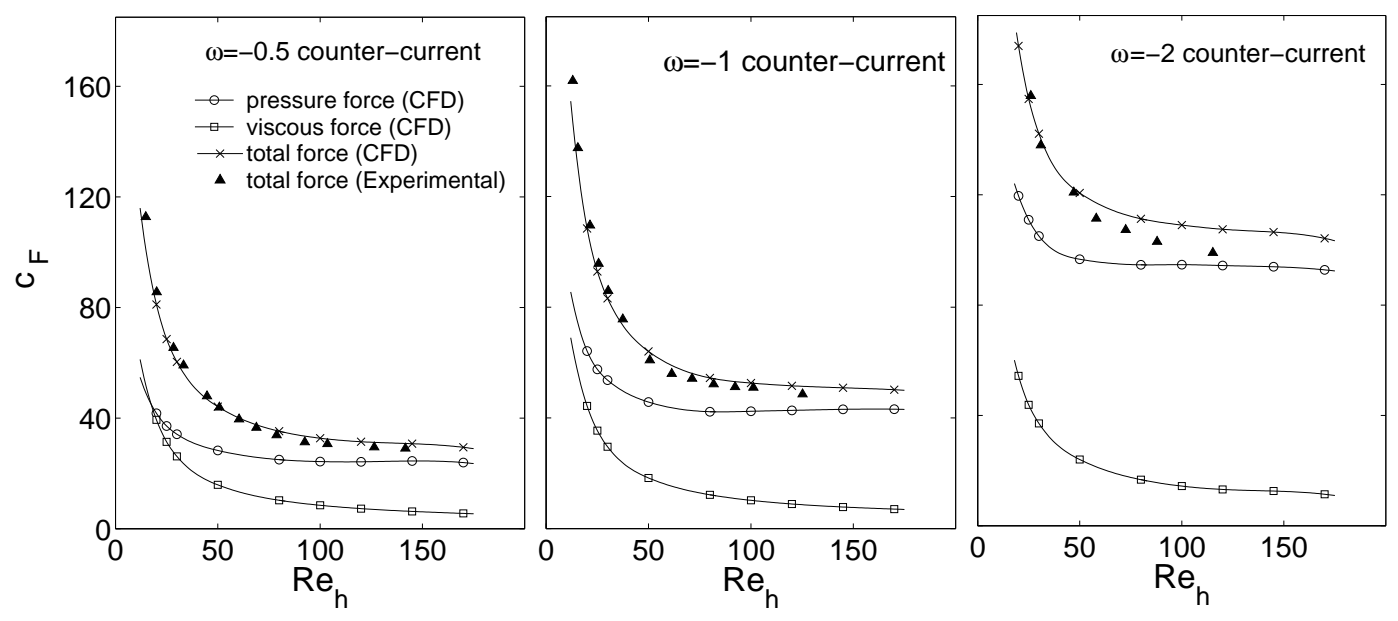



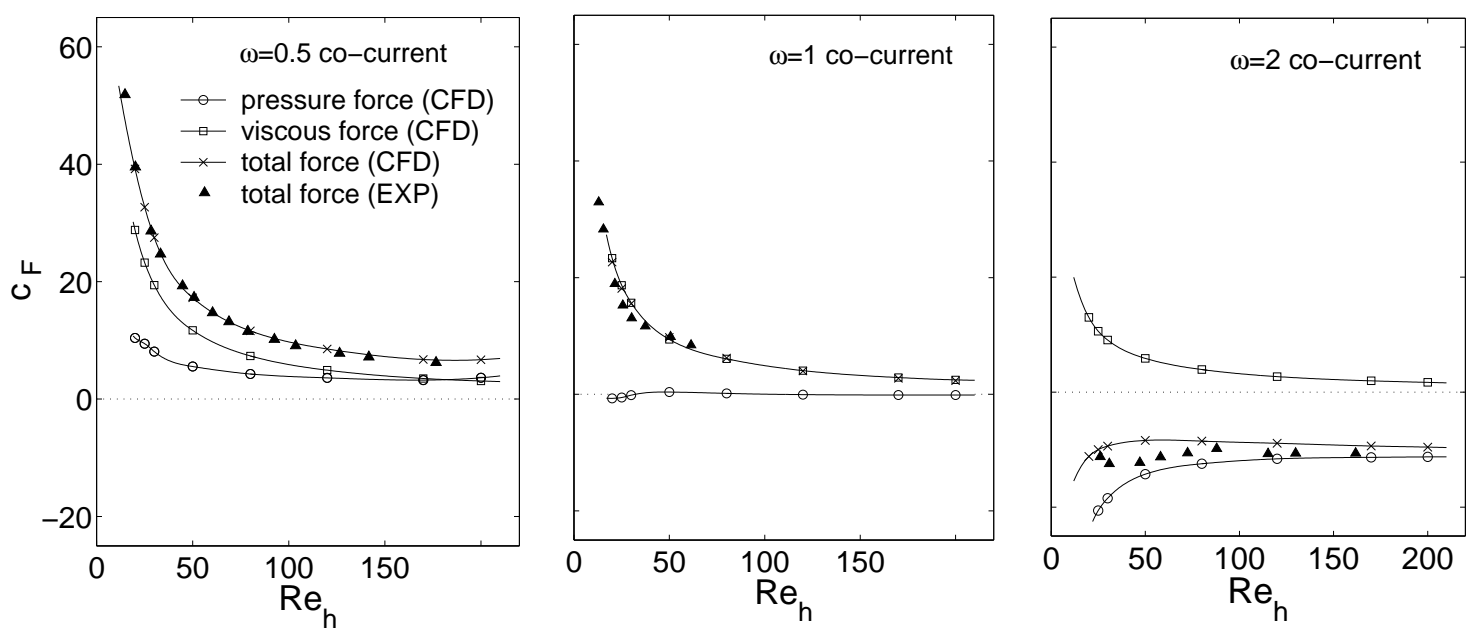